Aus dem pharmakol. Institut des Prof. S. O. Tschirwinsky in Jurjew (Dorpat).

\title{
Ueber \\ die Resorption und Ausscheidung des Eisens im Darmcanale der Meerschweinchen.
}

Von

Dr. med. G. Swirski, Assistenten am Institut.

(Hierzu Tafel VI.)

Es ist die Frage nach den Wegen der Aufnahme und Aus. scheidung des Eisens im thierischen Organismus noch nicht nach allen Richtungen hin als gelöst anzusehen, wenngleich die Bemühungen, auf chemischem Wege die Frage zu beantworten, durch Zuhülfenahme des Mikroskops sehr wesentlich unterstützt worden sind.

Wenn man in Betreff der Ausscheidung des sowohl per os, als auch subcutan einverleibten Eisens im Allgemeinen darin einig ist, dass dieselbe durch den Darm und die Nieren vor sich geht, so kann man dasselbe noch nicht bezüglich der Aufnahme des Eisens per os sagen. Es theilen sich in dieser Hinsicht die Autoren in zwei Lager: 1. in solche, die nur das Nahrungseisen, resp. organisch gebundenes Eisen dureh den Darm resorbirt wissen wollen; 2. in solche, die auch den anorganischen Eisenpräparaten eine Aufnahme durch den Darm vindiciren.

Die erste, von $\mathrm{Bung}^{1} \mathrm{e}^{\text {) }}$ aufgebrachte Anschauung, die nur dem Nahrungseisen den Durchtritt durch den Darm das Wort redet, für das medicamentös gereichte anorganische Eisen aber unter normalen Verhältnissen keine Resorption zugibt, hat in letzter Zeit viel Angriffe erfahren. Durch die Arbeiten von $\mathrm{Kunkel}^{2}$ ), Quincke ${ }^{3}$ ),

1) Bunge, Ueber die Assimilation des Eisens. Zeitschrift für physiol. Chemie Bd. 3 S. 49.1885.

2) Kunkel, Zur Frage der Eisenresorption. Pflüger's Archiv Bd. 50 S. 24. 1891 .

3) Quincke, Ueber Eisentherapie. Volkmann's Sammlung klin. Vorträge. Neue Folge Nr. 129. 1895. 
Ueber die Resorption und Ausscheidung des Eisens im Darmcanale etc. 467 Hall'), Woltering ${ }^{2}$ ), Gaule ${ }^{3}$ ), Macall $\mathrm{lm}^{4}$ ) und Anderen sind nicht ungewichtige Gründe gegen die Annahme vorgebracht, dass das medicamentös dargereichte Eisen bei intacter Darmschleimhaut nicht aufgenommen werde.

Fassen wir zunächst diejenigen Autoren dieser Gruppe in's Auge, welche den mikrochemischen Weg zur Lösung der Eisenfrage eingeschlagen haben, so findet mach $\mathrm{Hall}$, Hochhaus und Quincke die Aufnabme des Eisens ausschliesslich im Duodenum statt. Die genannten Forscher haben ihre Untersuchungen an Mäusen gemacht. Hochhaus und Quincke nehmen die Aufnahme des Nahrungseisens auch bei Meersehweinchen und Ratten im Duodenum als wahrscheinlich an.

Macallum kommt auf Grund seiner Untersuchungen an Meerschweinchen zum Schlusse, dass die Aufnahme des Eisens, falls geringe Fe-Mengen den Thieren verabfolgt werden, in dem, dem Pylorus anliegenden, wenige Zoll unfassenden Theile des Duodenum stattfindet; falls aber grössere Mengen auf ein Mal dem Thiere gegehen werden, findet die Resorption im ganzen Dünndarm statt.

Nach Gaule, dessen Versuchsthiere Kaninchen waren, geschieht die Resorption ausschliesslich im Duodenum, weder im Magen, noch im Dünndarm ist eine Resorption nachzuweisen. Nach ihm geht die Aufnahme durch die Darmepithelien und durch die centralen Lymphgefässe der Zotten vor sich, also in ähnlicher Weise wie bei dem Fette.

Was die Ausscheidung betrifft, so lässt $\mathrm{Hall}$ das Eisen durch die Galle, die Darmseerete und abgestorbenen Fpithelien aus dem Körper fortgeschafft werden. Hochhaus und Quincke lassen die Ausscheidung des Fisens bei Maus, Froseh, Kaninchen und Meerschweinchen durch die Schleimhäute des Coecums und Dickdarms vor sich gehen,

1) W. S. Hall, Ueber das Verhalten des Eisens zum Thierorganismus. Arch. für Anat. und Physiologie. Physiol. Abth. 1896 S. 49-84.

2) Woltering, Ueber die Resorbirbarkeit der Eisensalze. Zeitschrift für physiol. Chemie Bd. 21 S. 186.1895.

3) Gaule, Ueber den Modus der Resorption des Eisens und das Schicksal einiger Eisenverbindungen im Verdauungscanal. Deutsche medic. Wochenschrift 1896 Nr. 19 S. 289.

4) Macallum, On the absorption of iron in the animal body. The Journal of Physiology vol. 16 p. 268-297. Cambridge 1894. 
wobei die einzelnen Darmtheile je nach der Thierspecies in verschiedenem Grade an der Ausscheidung betheiligt sein sollen.

Nach diesen Autoren scheint die Ausscheidung in zeitlichen und örtlichen Schüben durch Auswanderung von Leukocyten und Abstossung von Epithelien stattzufinden. Bei Maus und Ratte nimmt auch die Niere an der Fe-Ausscheidung Theil.

Macallum lässt, bei Ueberfütterung des Organismus mit Eisen, dasselbe sowohl im Duodenum, als auch im Coecum und Dickdarm durch die Lieberkühn'schen Drüsen ausgeschieden werden. Leber und Nieren nehmen, nach diesem Autor, wenig Antheil an 'der Ausscheidung.

Den genannten Autoren zufolge geht die Resorption des Eisens im Duodenum vor sich. Hochhaus, Quincke und Hall; die an Mäusen experimentirten, wie auch Gaule, der Kaninchen untersuchte, stimmen hierin vollkommen überein.

Beim Meerschweinchen scheinen die Verhältnisse insofern anders zu liegen, als Macallum bei geringen Eisengaben, wie auch bei gewöhnlicher Fütterung im Duodenum allein die Aufnahme des Eisens vor sich gehen lässt; bei stärkeren Eisengaben aber, auf ein Mal gegeben; sollen, entsprechend der Menge, auch weitere Bezirke des Dünndarms an der Fe-Resorption sich betheiligen.

Es liegen in der Litteratur bis jetzt nur sehr wenige Mittheilungen über die Eisenreaction im Darmkanale bei Hungerzuständen vor. Macallum ist der einzige, der bei seinen Versuchen über die FeResorption Meerschweinchen hungern liess und dabei die Beobachtung machte, dass sich Fe-Reaction im Duodenum fand, obgleich sie eine Woche gehungert hatten. Er erklärt die Fe-Reaction als von der Galle herrührend, denn er beobachtete, dass, wenn er Thiere mit Eidotter, der nur sehr geringe Mengen locker gebundenes $\mathrm{Fe}$ enthält, fütterte, die Eisenreaction sehr schwach wurde; gab er nun den 'Thieren Aether, so strömte die Galle reichlich hervor, und die Reaction wurde so stark wie bei normalen Thieren. In diesem Verhalten findet er den Beweis dafür, dass die Fe-Reaction im Duodenum bei normalen Meerschweinchen auf der Resorption des Nahrungseisens beruht und, was im Hungerzustande von Fe-Reaction noch vorlag, aus der Galle stammte.

Nach den Untersuchungen von A. Lipski ${ }^{1}$ ) ist die Eisen-

1) A. Lipski, Ueber die Ablagerung und Ausscheidung des Eisens aus dem thierischen Organismus. Arbeiten d. Pharmak. Instituts zu Dorpat Bd. 9 S. 78. 
reaction im Duodenum hart unterhalb des Pylorus der Frösche (ein ca. $1 \mathrm{~cm}$ umfassender Abschnitt des Duodenums) constant vorhanden, sie ist als "physiologische Siderosis" anzusehen. Sie unterbleibt nicht, wie auch ich mich habe äberzeugen können, nach Unterbindung des Ductus choledochus dieser Thiere, auch nicht, wenn letztere längere Zeit nur in destillirtem Wasser gehalten worden waren.

Diese Erscheinung ist dem Frosche so eigenthümlich, dass man sie bei jedem Individuum findet. Bei den Kröten fand ich ähnliche Verhältnisse, während Schildkröten und Salamander dieses Verhalten nicht zeigten.

Die Betrachtung eines mikroskopischen Sehnittes aus dem genannten Theile des Duodenums beim Frosche zeigt die Interstitien der Epithelien angefült mit rundlichen Körnchen, die ohne jede Behandlung bräunlich, bei $\left(\mathrm{NH}_{4}\right)_{2} \mathrm{~S}-$ Behandlung schwarzgrün, bei Ferrocyankalium $+\mathrm{HCl}$ schön blau erscheinen. Die Epithelzellen selbst zeigen keine Reaction, nur hier und da erscheint das Protoplasma in der Nähe der stark mit Fe-Körnern angefüllten Interstitien diffus blau gefärbt. Unter dem Epithel sieht man riesige Leukocyten, angefüllt mit Körnehen von der Beschaffenheit, wie sie oben besehrieben wurde.

Dass diese Fe-haltigen Leukocyten die Tendenz haben, aus dem Bindegewebe zum Darmlumen hinzuwandern, sieht man daraus, dass viele von ihnen zwisehen den Epithelzellen sich befinden, während andere schon ausserhalb des Epithels in's Darmlumen gelangt sind. Wenn ich jetzt vorgreifend bemerke, dass für die Resorption dieses Bild nicht spricht, so ist dasselbe mit Berücksichtigung des Umstandes, dass es bei Hunger, wie auch nach Unterbindung des Ductus choledochus nicht ausbleibt, als ein Ausscheidungsvorgang anzusehen.

Es hätte nun, auf Grund des soeben erwähnten Befundes bei Fröschen, die trotz Hungerns bestehen bleibende Fe-Reaction im Duodenum der Meerschweinchen als auf Ausscheidung aus dem Organismus beruhend gedeutet werden können. Daher stellte ich mir die Aufgabe, diese Verhältnisse bei den Meersehweinchen einer näheren Prüfung zu unterziehen. Diese Frage bildete den Ausgangspunkt meiner Arbeit, an deren Beantwortung ich mich folgendermaassen machte. Da nämlich die Autoren ihr Augenmerk hauptsächlich auf die Aufnahme von dargereichten Fisenpräparaten gerichtet hatten, Hungerzustände aber nur von $\mathrm{Macallum}$ in nicht eingehender Weise untersucht waren, so hoffte ich, durch Berück- 
sichtigung dieser Seite vielleicht eine Klärung der Frage herbeizuführen.

Nachdem ich zuerst die normalen Verhältnisse der Duodenalschleimhaut untersucht hatte, fütterte ich zwei Meerschweinchen mit Fe-armer Nahrung, um den Einfluss des Ausfalles der Eisenzufuhr auf den Eisengehalt der Duodenalschleimhaut zu studiren. Ausserdem wurden zwei Meerschweinchen der absoluten Carenz ausgesetzt. Das Ergebniss war, dass die Fe-Reaction in den Epithelien des Duodenums schwand, die Leukocyten aber immer Fe-Reaction aufwiesen. Jetzt konnte der Kinwurf gemacht werden, diese Fe-Reaction käme von der Galle. Daher unterband ich den Ductus choledochus; aber auch jetzt war die Reaction nicht geschwunden. Die Annahme, dass die bei Hungerzuständen vorhandene Fe-Reaction von der Galle herrühre, war hierdurch widerlegt worden.

Es handelte sich jetzt darum, zu erklären, woher die Fe-Reaction der Leukocyten stammte. Um dieser Frage näher zu kommen, fand ich in der Beobachtung, dass das wegen Unterbindung des Ductus choledochus operirte Meerschweinchen, welches auf absolute Carenz gesetzt war, trotz aller Vorsichtsmaassregeln am Kothfressen nicht verhindert werden konnte, eine willkommene Stütze. Ich überzengte mich davon, dass eine sorgfältige Entfernung des Kothes, wie auch das Aufstellen der Thiere auf Drahtgitter, wie Rubner ${ }^{1}$ ) es bei Kaninchen that, absolut ungenügend ist. Das Meerschweinchen, von Hunger gepeinigt, erfasst eben den Koth, bevor er zu Boden fällt, und verzehrt ihn. Daher entschloss ich mich, den Meerschweinchen einen Maulkorb aufzusetzen, um das Verhalten der Fe-Reaction im Dünndarme bei vollkommenem Ausschlusse des Kothfressens zu beobachten.

Das Resultat dieser Untersuehungsreihe war, dass gradatim die Fe-Reaction, gebunden an Leukocyten, abnahm und nach einem Hunger von $5 \times 24$ Stunden ganz verschwunden war. Und zwar waren auch die Leukocyten selbst geschwunden. Hierdurch war der Beweis erbracht, dass der Koth bei absoluter Carenz der Meerschweinchen ohne Maulkorb die Fe-Reaction im Duodenum, gebunden an Leukocyten, vermittle.

1) Rubner, Ueber den Stoffwechsel im hungernden Pflanzenfresser. Zeitschrift für Biologie Bd. 17 S. 216. 1881. 
Ueber die Resorption und Ausscheidung des Eisens im Darmcanale etc. 471

Zum Schluss fütterte ich einige Meerschweinchen mit Fe-Präparaten, um die Verhältrisse im Darmcanale unter diesen Umständen kennen zu lernen.

\section{Untersuchungsmethoden.}

Die Thiere wurden gewöhnlich durch Entbluten getödtet, indem ihnen die Halsgefässe durch einen Schnitt eröffnet wurden. Finigen wurde in der Chloroformnarkose die Bauchhöhle blossgelegt und lebenswarm die einzelnen Theile des Darmes entnommen. Je nach dem Zwecke gelangten die Darmstücke entweder zusammen mit dem Inhalte oder aufoeschnitten nach Abspülung in destillirtem Wasser, physiologischer NaCl-Lösıng oder auch Leitungswasser in Formalinlösung ( $4 \%$ Formalin in physiologischer Kochsalzlösung), wo sie 24 Stunden verblieben. Zur makroskopischen Reaction kam weder $\mathrm{zu}$ altes, noch $\mathrm{zu}$ frisches $\left(\mathrm{NH}_{4}\right)_{2} \mathrm{~S}$ des Laboratoriums in Anwendung; von so behandelten Stücken wurden Zupfpräparate oder abgeschnittene Theilchen sofort mikroskopisch untersucht. Aus der Formalinlösung kamen die Theile in $96 \%$ Alkohol. Die Uebertragung in absoluten Alkohol gab mir beim Meerschweinchen nicht so gute Resultate. Die Einbettung geschah für gewöhnlich in Celloidin. - Zu feineren Schnitten kamen die Präparate in Paraffin. Zu Dauerpräparaten wurden die Schnitte benutzt, die die Berlinerblau-Reaction durchgemacht hatten. Zu letzterem Behufe kamen die Schnitte, nachdem sie vou Alkohol befreit worden, auf 10 Minuten bis zu einer halben Stunde in $1,5 \%$ Ferrocyankaliumlösung, von da auf ca. 1-2 Minuten in eine $0,45 \%$ Salzsäurelösung. Nach Abspülen in destillirtem Wasser wurden sie in Alaun-Carmin gefärbt, durch Origanum-Oel aufgehellt und in Canadabalsam eingeschlossen. Zur Besehreibung der Präparate wurde jedesmal ein Schnitt in $\left(\mathrm{NH}_{4}\right)_{2} \mathrm{~S}$ auf 5 Minuten ungefähr, je nach der Dicke desselben, getancht und in Glycerin untersucht.

Es diente das zur Controle des Berlinerblau-Präparates, wobei ich mich überzeugen konnte, dass der Unterschied im Ganzen unbedeutend ist, und dass man bei einiger Uebung sehr bald Kunstproducte erkennen lernt.

Jedenfalls habe ich bei meinen sehr zahlreichen Untersuchungen an den Meerschweinchen kein einziges Mal Verhältnisse gefunden, die denen glichen, wie Q u in ck ${ }^{1}$ ) sie am Froschmagen beobachtet hat.

1) Quincke, Ueber directe Fisenreaction in thierischen Geweben. Airchiv für experim. Pathol. und Pharm. Bd. 37 S. 189. 1896. 
Die Turnbullsblau-Reaction ${ }^{1}$ ) habe ich auch dazwischen angewandt und sehr intensive und extensive Färbungen bekommen, die wohl auf wirksam gewordene oxydulische Eisenpartieen zurückzuführen sind. Die feineren Schnitte litten aber so sehr bei dieser Behandlung, dass ich nur sehr beschränkten Gebrauch von ihr gemacht habe. Bei den Manipulationen in $\left(\mathrm{NH}_{4}\right)_{2} \mathrm{~S}$, wie auch bei Ferrocyan-

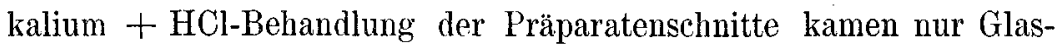
nadeln und Knochenpincetten in Anwendung.

Finen Versuch machte ich noch, die in $\left(\mathrm{NH}_{4}\right)_{2} \mathrm{~S}$ gelegten Darmstücke, auf Cartonpapier geklebt, zu conserviren. Sie kleben ausgezeichnet an und erhalten nach 24 Stunden einen schönen Glanz, wie wenn die Theile mit Lack überzogen wären. Wenngleich die aufgeklebten Stücke sehr bald abblassen, so erhält man doch das Relative sehr gut, und man kann noch nach einem halben Jahre sehr deutlich die Unterschiede zwischen den einzelnen Darmtheilen feststellen. Das $\left(\mathrm{NH}_{4}\right)_{2} \mathrm{~S}$ darf hierbei auf keinen Fall älter als zwei Wochen sein, sonst stört der ausgefällte Schwefel das Bild erheblich. Man kann sich dann noch dadurch helfen, dass man mit einem Wattebausch das Ueberschüssige leicht entfernt. Zu Demonstrationszwecken dürfte dieser Modus verwertet werden können.

\section{Normale Meerschweinchen.}

Um die Eisenreaction im Darmkanale der Meerschweinchen unter normalen Verhältnissen zu studiren, unterzog ich drei männliche Thiere der Untersuchung und zwar zu verschiedenen Zeiten. Das erste (I), im October 1896 untersuchte war mit Schwarzbrot, Kohlblättern und Kartoffeln gefüttert worden, 2 Jahre alt und $625 \mathrm{~g}$ schwer. Das im März 1897 entblutete (II) wog $340 \mathrm{~g}$, war ein halbes Jahr alt und wie das vorige ernährt. Das dritte (III), im September 1897 getödtete, 9 Monate alte war $482 \mathrm{~g}$ schwer und hatte fast ausschliesslich Grünfutter erhalten.

Magen. Makroskopisch wird er grün bei allen drei Meerschweincben unter $\left(\mathrm{NH}_{4}\right)_{2} \mathrm{~S}$-Behandlung. Der Pylorustheil ist etwas dunkler gefärbt als die pars fundi ventric. Mikroskopisch lässt sich keine Fe-Reaction nachweisen.

1) Joh. Tirmann, Ueber Eisenablagerung S. 11. Diss. Jurjeff, 1896. 
Du o d enum. Die Strecke rom Pylorus bis zum Coecum, theilte ich in 3 gleiche Theile; es entsprach diese Eintheilung ziemlich genau dem charakteristischen Verhalten der Theile. Auf das Duodenum kamen hierbei ungefähr $33 \mathrm{~cm}$, von welchen die oralwärts belegenen 10-15 cm eine deutliche Fe-Reaction makroskopisch aufwiesen. Bei dem ausschliesslich mit Gras ernährten Thiere war die Reaction etwas schwächer. Diese im oberen Drittel des Duodenums bei $\left(\mathrm{NH}_{4}\right)_{2} \mathrm{~S}$ Behandlung auftretende Dunkelgrün-Färbung, mit einem Anfluge in's Schwärzliche, wird analwärts schwächer, um im unteren Theile ganz zu verschwinden.

Stellt man sich aus einem so behandelten Duodenalschleimhautstücke ein Zupfpräparat her, so sieht man unter dem Mikroskope grosse Leukocyten, grünschwarz verfärbt, dann Schollen und feinere Körnchen im Stromagewebe, wahrscheinlich von zerfallenen Fe-haltigen Leukocyten herrührend, und drittens grünlich verfärbte Epithelzellen, die feine schwarze Körnchen enthalten.

Bekommt man das mosaikartige Peripheriebild der Epithelzellen zu sehen, so überzeugt man sich, dass die feinen schwarzen Körnchen in dem Protoplasma der einzelnen Zellen selbst eingelagert sind. Auf mikroskopischen Schmitten lässt sich constatiren, dass diese Eisenablagerung in feinen Partikeln, nicht in allen Zotten vorkommt. Wo man sie aber sieht, da nimmt sie, wie Hochhaus und Quincke es angegeben haben, meistens das äussere Viertel der Zelle ein reicht nie bis zum Kern und ist im Basalsaume nie anzutreffen. Solehe Epithelzellen finden sich öter an den Seitentheilen der Zotten, als an der Zottenspitze. Bei Anwendung von Immersionssystemen constatirt man ein amorphes Pulver, das aus gröberen und feineren Körnchen zusammengesetzt ist.

Im Stroma der Zottenspitze sieht man ganz besonders reichlich Fe-Reaction aufweisende Leukocyten. Sie stellen grosse Zellen dar, die bei Alaun-Carminfärbung nach vorheriger Behandlung mit Ferrocyankalium + HCl einen schön roth gefärbten Kern wahrnehmen lassen. - In dem Protoplasma finden sich die feinen Körnchen, blaugefärbt, eingelagert. Ausser dem Kerne findet man noch hier und da Einlagerungen in dem Protoplasma, die keine Fisenreaction geben. Von dieser grossen Form abgesehen, findet man noch Fe-Reaction aufweisende Zellen kleiner Form, wo das Protoplasma eine diffuse Reaction zeigt und der Kern auch roth gefärbt ist. Ferner erblickt man solche, die nur eine runde, blane Scheibe darstellen oder bei 
$\left(\mathrm{NH}_{4}\right)_{2} \mathrm{~S}$-Behandlung grünschwarz erscheinen. Ob das ein protoplasmaarmes Gebilde, also ein Fe-Reaction gebender Kern ist oder eine Zelle mit verschwindend kleinem Kern, konnte ich nicht entscheiden.

Die grossen Formen entsprechen genau den Zellen, die H eid en ha in ${ }^{1}$ ) beim Meerschweinchen zuerst genauer beschrieben hat, nachdem sie schon Heitzmann's ${ }^{2}$ ) Aufmerksamkeit auf sich gelenkt hatten.

Es sind das die dem Meerschweinchen eigenthümlichen Phagocyten. An manchen Stellen sieht man die grosse Form der Leukocyten in den Gefässen, die den centralen Chyluscanal begrenzen, bis zu der Basis der Zotte hin.

Jejunum. Dasselbe zeigt an keinem der drei Thiere FeReaction. Namentlich fehlt die feine Ablagerung in den Epithelzellen, wie auch die Fe-Reaction gebenden Phagocyten. Makroskopisch war das Jejunum der beiden mit Brot und Kartoffelschalen gefütterten Thiere dunkler, als das bei dem mit Gras ernährten.

Il e um. Nur bei Meerschweinchen II liessen sich $\mathrm{Fe}-\mathrm{Cyten}^{3}$ ) nachweisen, trotzdem makroskopisch gerade bei den beiden anderen Thieren die Fe-Reaction stärker war. Im Epithel keine Ablagerung.

Coecum. Dieser Darmtheil, der stets prall gefüllt mit Inhalt sich präsentirt und selbst bei Thieren, die an absoluter Carenz eingegangen sind, nie leer wird, hat, von seinem Inhalte sorgfältig abgewaschen, ein bräunliches Colorit. Ein ungefärbter Schnitt aus dieser Gegend zeigt in dem interglandulären Gewebe die Pbagocyten von gelb-bräunlicher Farbe. Behandelt man es mit $\left(\mathrm{NH}_{4}\right)_{2} \mathrm{~S}$, so nehmen die genannten Gebilde eine grünschwarze Färbung an. Wird nun ein frisches Darmstïck in $\left(\mathrm{NH}_{4}\right)_{2} \mathrm{~S}$ getaucht, so wird dasselbe in einigen Secunden kohlschwarz. Ein Zupfpräparat davon unter das Mikroskop gebracht, zeigte die schon von Hochhaus und Quincke beobachtete netzförmige Anordnung des durch die $\mathrm{Fe}$ -

1) R. Heidenhain, Beiträge zur Histologie u. Physiologie der Dünndarmschleimhaut. Pflüger's Archiv Bd. 43 S. 41. Suppl. 1888.

2) C. Heitzmann, Zur Geschichte der Dünndarmzotten. Sitzungsberichte der Wiener Alrademie Bd. 58, Sitzung vom 23. Juli 1868.

3) Der Kürze wegen werde ich im Folgenden die Leukocyten und Phagocyten, wenn sie mit den entsprechenden Reagentien behandelt Fe-Reaction geben, "Fe-Cyten" nennen. 
Ueber die Resorption und Ausscheidung des Eisens im Darmcanale etc. 475

Reaction scharf von den Drüsenzellen sich abhebenden interglandulären Bindegewebes. In den Epithelzellen keine Fe-Ablagerung, wie das im Duodenum der Fall war.

Ein ganz besonders auffallendes Object war das Coecum vom Meerschweinchen I. Der unbehandelte Präparatenschnitt zeigte einen aus dem Lumen der Lieberkühn'schen Drüse hervorquellenden Inhalt, der zum Darmlumen hin stärker, zum Fundus der Drüse hin allmählich abnehmend, bräunlich verfärbt ist. Die Farbe stimmt vollkommen mit der bräunlichen der im periglandulären Bindegewebe befindlichen Leukocyten überein. Mit $\left(\mathrm{NH}_{4}\right)_{2} \mathrm{~S}$ behandelt, werden alle bräunlichen Partieen schwarz, resp. braun, die Zellen im interglandulären Gewebe sowohl, als auch die aus dem Lumen der Krypten hervortretenden Pfröpfe. Auf Schnitten, an denen der Darminhalt mit gehärtet worden war, findet man im selben eine Menge schon entleerter ausgestossener Pfröpfe, die meistens eine sehr starke FeReaction aufwiesen. Bei der Berlinerblau-Reaction lässt sich an den Gebilden, unter Benutzung von Immersionssystemen, eine feine Granulirung wahrnchmen, aber sonst keine Andeutung einer besonderen Structur erkennen.

Zwischen dem hyalinen Basalsaume der Kryptenzellen und dem Pfropfe ist oft ein Zwischenraum durch Schrumpfung des letzteren entstanden. Im Fundus ist die Fe-Reaction sehr gering, bisweilen fehlt sie ganz, dann nimmt sie allmählich zur Mitte der Drüse hin zu, un dann am Ausgang der Drüse plötzlich sehr stark zn werden. Die am Ausgange der Drüse befindlichen Theile der Pfröpfe sind ebenso stark tingirt wie die schon im Darmlumen befindlichen. Auf dem Drüsenquersehnitte sieht man einen centralen blauen Punkt, umgeben von einem hellen hyalinen Rande, dem Basalsaume der Drüsenzellen. Die Drüsenzellen selbst zeigen niemals eine Fe-Reaction, weder in körniger, noch in diffuser Form. Dass die besprochenen Pfröpfe nicht aus dem Darmeanale stammen, sondern eine consistentere Form des Mucins der Lieberkühn'schen Drusen darstellen, ist nach dem oben gesagten wohl unzweifelhaft. Hinzufügen möchte ich noch, dass die genannten Pfröpfe auf jedem Schnitte aus dem Coecum des Thieres I zu sehen waren, somit also im ganzen Coecum vertreten sein mussten, da ich aus den verschiedensten Theilen desselben Schnitte verfertigt habe. Es ist das ein seltener Befund, wenngleich man einzelnen Pfröpfen hier und da schon begegnet. Bei Meerschweinchen II und III konnte ich nichts Aehnliches constatiren. 
Das interglanduläre Bindegewebe ist bei allen dreien stark mit Fe-Cyten angefüllt, aber auch in der Submucosa, sowohl in den Interstitien des febrillären Bindegewebes, wie auch in der Umgebung der grossen Gefässe sind mächtige Fe-Cyten anzutreffen.

Die Zellen der Lieberkühn'schen Drüsen sind frei von jeglicher Fe-Reaction, wovon man sich auf Längs-, wie auf Quersehnitten überzeugen kann. Das Oberflächenepithel hingegen zeigt deutliche $\mathrm{Fe}-$ Reaction beim Meerschweinchen II. Es bezieht sich das nur auf die mittlere Zellengruppe, die so angeordnet ist, dass durch ein Auseinanderweichen der Zellen eine Art Trichter gebildet wird. Die breite Seite dieses Trichters ist zum Darmlumen hingewandt. Die Epithelzellen zeigen hier eine diffuse Fe-Reaction, wovon man sich auch durch $\left(\mathrm{NH}_{4}\right)_{2}$ S-Behandlung überzeugen kann. Die Zwischensubstanz der Zellen zeigt ebenfalls eine diffuse Fe-Reaction. Man erhält den Eindruck, als wenn eine Fe-haltige Flüssigkeit durch diese Zellenpartie hindurchginge, das interglanduläre Bindegewebe somit vermittelst der Oberfächenepithelien mit dem Darmlumen in Verbindung trete.

Ob die Richtung zum Darmcanale hin ging oder a us demselben zum adenoiden Bindegewebe hin, konnte aus dem Bilde nicht entschieden werden. - Die Fe-Cyten, die im Stroma des interglandulären Gewebes ziemlich zahlreich angetroffen werden, sind von grosser und kleiner Form. An der grossen Zellenform, die wir beim Duodenum kennen gelernt haben, kann man einen roth gefärbten Kern mit Kernkörperchen deutlich unterscheiden.

Das Protoplasma selbst ist hellrosa gefärbt, die eingelagerten Körnchen sind bei der Ferrocyankalium + HCl-Färbung auf dünnen Schnitten grünbläulich.

Es ist nach dem Gesagten sicher, dass die Lieberkühn'schen Krypten, entgegen der Anschauung von $\mathrm{H}$ oppe-Seyler, keine aufnehmenden, sondern ausscheidende Apparate sind.

Andererseits erscheint es wahrscheinlich, dass das Oberflächenepithel entweder an der Aufsaugung oder Ausscheidung sich betheiligt, und zwar nicht allein durch die Zellen selbst hindurch, sondern auch durch die Interstitien derselben.

Im oberen und unteren Dickdarm ist makroskopisch die Reaction viel schwächer als im Coecum. Es ist die Masse der Leukocyten mit Fe-Reaction geringer, daher erscheinen auch die 
Falten der Schleimhaut an sich mehr scbiefergrau, als so gesättigt schwarz, wie es im Coecum der Fall war.

Mikroskopisch sieht man im interstitiellen Gewebe ganz dieselben Fe-Cyten grosser und kleiner Form, nur nicht so zahlreich wie im Coecum. An den Epithelzellen ist keine Fe-Reaction wahrzunehmen. Es lassen sich in diesen Darmtheilen keine Zeichen für eine Resorption an dem Oberflächenepithel nachweisen. Schleimzellen sind im unteren Theile des Dickdarmes hänfiger anzutreffen als im oberen.

Peyersche Haufen geben makroskopisch durchweg schwarzgrüne Reaction. Mikroskopisch findet man bei Meerschweinchen II nur hier und da in der Peripherie eines solitären Follikels einen Fe-Cyt vor, und zwar an der vom Darmlumen abgewandten Partie. Es steht das nicht im Verhältniss zum makroskopischen Befunde. Man muss sich das Fe-haltige Material in einer so feinen Vertheilung vorstellen, dass es der mikroskopischen Wahrnehmung entgeht.

Leber. Makroskopisch liess sich eine dunkelgrüne Färbung der Lebersubstanz nach $\left(\mathrm{NH}_{4}\right)_{2} \mathrm{~S}$ nachweisen. Es war aber eine diffuse, mehr makroskopisch sich manifestirende Reaction. Mikroskopisch war bei keinem der normalen Thiere eine Fe-Reaction weder in den Capillaren der Leber, noch in den Zellen zu constatiren.

Milz. Makroskopisch wurde das ganze Organ violett-schwarz. Mikroskopisch findet sich Fe-Reaction in der Pulpa, und zwar sind es die Lymphsinus, in denen man freiliegende Schollen, grosse und kleine Formen von Fe-Cyten antrifft. Diffuse Fe-Reaction finde ich in der nächsten Ungebung der Malpighi'schen Körperchen. Fs findet sich das Fe daselbst wahrscheinlich in Iösung and unspült so die in der Bildung begriffenen Leukocyten.

Die Untersuchung der normalen Meerschweinchen ergibt also, entsprechend den Untersuchungen von Hochhaus und Quincke und Macallum, eine Fe-Reaction im Duodenum, und zwar in zweierlei Form, die eine in feinen Partikeln in den Epithelzellen selbst, die andere gebunden an Leukocyten. Die erstere Reaction, also die Ablagerung in feinen Partikeln, war nur im Duodenum zu finden. Im Jejunum fanden wir keine solche Fe-Reaction. Im Ileum liess sich bei einem Thiere eine Fe-Reaction, gebunden an Leukocyten, deutlich nachweisen, bei z we i en war keine Fe-Reaction. Das Coecum zeigte ein Bild, das auf den ersten Blick für Ausscheidung des Eisens in Form von Mucinpfröpfehen sprach. 
In Betreff dieser Erscheinung bin ich nach reiflicher Ueberlegung zum Schluss gekommen, dass sie, entgegen der Annahme von Macallum, keine Ausscheidung des Eisens aus dem Organismus darstellt, sondern ein an die Eigenthümlichkeiten des Mucins gebundener Vorgang von Aufsaugung des Eisens aus dem Darmcanale ist.

\section{Meerschweinchen mit Fe-armem Futter ernährt.}

Nachdem Hochbaus und Quincke ${ }^{1}$ ) die Beobachtung gemacht hatten, dass normale Meerschweinchen Fe-Reaction im Duodenum zeigten, niusste es von Interesse sein, diesen Darmtheil unter Umständen zu untersuchen, wo die Fe-Zufuhr in der Nahrung vermieden, resp. vermindert war. Die Arbeit von W. S. Hall ${ }^{2}$ ) war mir, als ich meine Versuche begann, nicht bekannt, sonst hätte ich mich bestimmt dazu entschlossen, nach seiner Angabe Fe-freies Futter zu bereiten.

Nachdem ich zwei Combinationen von Fe-freien Nährstoffen vergeblich den Thieren vorgelegt hatte, gab ich ihnen Prince-AlbertCakes (aus der Fabrik Landrin), wovon sie sofort etwas nahmen. Die qualitative Untersuchung dieses Gebäckes ergab geringe $\mathrm{Fe}$ Reaction. Eine nachträglich angestellte quantitative Analyse ergab $0,0029 \% \mathrm{Fe}_{2} \mathrm{O}_{3}$, während Kubmilch 0,003 , Kartoffeln 0,042 und Eidotter $0,040 \% \mathrm{Fe}_{2} \mathrm{O}_{3}$ in der Trockensubstanz aufweisen ${ }^{3}$ ).

Im October 1896 wurden 2 Meerschweinchen männlichen Geschlechtes unter je eine Glasglocke gesetzt, welche auf einer grossen Glasplatte stand. Die Glocke war an ihrem oberen Theile mit einer grossen Deffnung für den Durchtritt der Luft versehen. Ein Schälchen dest. $\mathrm{H}_{2} \mathrm{O}$ war gegen den Durst hingestellt. Die äusseren Erscheinungen, die während der Versuche an den Thieren zu beobachten waren, stimmten im Ganzen mit dem Bilde überein, wie es von Lukjano ${ }^{4}$ ) für die Meerschweinchen bei absoluter Carenz

1) Hochhaus und Quincke, Ueber Fe-Resorption und Ausscheidung im Darmcanale. Arch. f. exper. Pathol. n. Pharm. Bd. 37 S. 174.

2) Hall, Einige Bemerkungen über dic Herstellung eines künstlichen Futters. Du Bois-Reymond's Archiv 1896 S. 142.

3) Bunge, Lehrbuch der phys. und path. Chemie. 3. Aufl. S. 100. 1894.

4) Lukjanow, cit. nach Lasarew, Zur Lehre von den Veränderungen des Gewichtes und der Zellenelemente einiger Organe und Gewebe bei den verschiedenen Perioden des abs. Hungerns. Inaug.-Diss. Warschau 1895 (russisch). 
beschrieben wird. Lukjanow unterscheidet vier klinische Bilder oder Perioden bei der absoluten Carenz: 1. das indifferente Stadium, 2. das Stadium der Excitation, 3. das Stadium der Depression und 4. das Stadium der Paralysen. Nachdem meine Meerschweinchen, die bis dahin in Gesellschaft mit anderen gehalten worden waren, sich an den neuen beengten Raum einigermaassen gewöhnt hatten, frassen sie an dem ersten Tag anscheinend gern von dem vorgelegten Futter. Aber schon gegen Ende des zweiten Tages sah man sie den Koth fressen, was sie sonst nicht thun, wenn ihnen das Futter convenirt. Eisenfreies Filtrirpapier nagten sie mit Begier, nahmen wieder etwas von der Nahrung und verhielten sich verhältnissmässig ruhig bis zum 4. Tage. Von da ab wurden sie sehr unruhig, liefen viel längs der Glaswand her, um einen Ausweg zu finden, nagten etwas von der Nahrung, berührten das täglich frisch hingestellte Wasser aber nicht. Dieses dauerte einen bis zwei Tage und würde dem Excitationsstarium entsprechen. Darauf wurden sie ruhiger, knäuelten sich zu einer Kugel, bekamen ein struppiges Aussehen und schienen zu schlafen. Das Mcerschweinchen $\mathrm{B}$, das jüngere von beiden, berührte von jetzt ab das Futter nicht mehr, während das andere dazwischen noch an den Prince-Albert-Cakes nagte. Dieses Depressionsstadium ging schon am 8 . Tage bei Meersehweinchen B in das der Paralyse über; es schwankte beim Gehen, glitt auf dem glatten Glasboden leicht aus und war nicht mehr im Stande, sich aufzurichten. Es lag dann ruhig auf der Seite und machte, sobald man sich ihm näherte, fruchtlose Bewegungen sich aufzurichten. Die Respiration wurde oberflächlich und beschleunigt. Da das Ende schon nahe schien, so kam es in diesem Stadium zur Entblutung. Das Meerschweinchen A, das 3 Monate älter war, nagte noch bis zum 11. 'Tage an dem Brote, worauf es entblutet wurde, bevor das paralytische Stadium eintrat.

Meerschweinchen $B$.

Durch Entbluten wird es am 8. Tage der Fütterung mit Fearmer Nahrung getödtet.

Section. Der Magen ist durch Gase aufgetrieben und enthält bräunliche Krümel, von denen einige der Schleimhaut sehr fest anhaften. Das D u od enum enthält keinen consistenteren Inbalt, ist aber gashaltig. Im Jejunum kleine Kothballen, ebenso im Ileum. Das Coecum ist stark durch Gase dilatirt und enthält chokoladenE. Pflüger, Archiv für Physiologie. Bd. 74 . 
braun gefärbte Kothmassen von verhältnissmässig bedeutender Quantität. Die Schleimhaut des ganzen Darmtractus ist äusserst blass. Mit $\left(\mathrm{NH}_{4}\right)_{2} \mathrm{~S}$ behandelte Stücke vom $\mathrm{M}$ age $\mathrm{n}$ zeigten grünliche Färbung; im Duodenum war deutliche Fe-Reaction fast wie beim normalen Thiere, im Jejunum sehr schwache, im Ileum wieder etwas stärkere. Unter dem Mikroskope sah man am frischen, mit $\left(\mathrm{NH}_{4}\right)_{2} \mathrm{~S}$ behandelten Stücke vom Duodenum deutliche Fe-Cyten unter dem Epithel. Das Coecum wird dunkel schwarzgrün und zeigt unter dem Mikroskope die netzartige Anordnung des interglandulären Gewebes.

Mikroskopisches. Im Magen lässt sich nicht eine Spur von Fe-Reaction nachweisen. Keine Hyperämie der intacten Schleimhaut. In der Pars pylorica ventr. findet man braune, an den Rändern gelblich durchscheinende Stückchen, die dem Epithel der Drüsen innig anhaften. Es sind das aus dem gefressenen Koth stammende Theile.

Du odenum. Fe-Ablagerung in feinkörniger Form wie bei normalen Thieren ist hier am Duodenum nirgends zu bemerken. Fe-Cyten grosser Form sind in dem Stroma der Zotten im oberen Theil des Duodenums sehr schön zu sehen. An manchen Zotten sieht man sie ganz im Epithel an der Spitze sitzen, wahrscheinlich im Begriffe, die Zotten zu verlassen, denn ausserhalb im Darmlumen sind schon Fe-Cyten befindlich und zwar von ganz gleicher Form. Das Epithel der Zotten ist überall gut erhalten und erscheint nirgends pathologisch verändert.

Coecum. Im interglandulären Gewebe stösst man auf reichlich anwesende Fe-Cyten grosser und kleiner Form. Ausserdem findet man hier in den Lumina der Lieberkühn'schen Krypten nicht selten blaue Pfröpfe, die zum Darmlumen hinausgestossen werden. Bei genauerer Betrachiung machen die in den Krypten steckenden Pfröpfe den Eindruck, als seien sie aus einzelnen Stücken zusammengesetzt. Fine Structur kann man bei starker Vergrösserung in ihnen nicht erkennen. Ebensowenig ergiebt die Durchmusterung der Querschnitte der Lumina einen Anhaltspunkt für eine Einwanderung von Fe-Cyten in die Krypten. Man sieht schon hier und da Trichterbildungen an dem Oberfächenepithel, durch welche Leukocyten und Erythrocyten in. ziemlich grosser Menge hindurchschlüpfen; viele Erythrocyten findet man im Darmlumen, dicht am Epithel. In der Submucosa sind eigentlich gar keine Fe-Cyten zu 
Ueber die Resorption und Ausscheidung des Eisens im Darmcanale etc. 481 treffen gewesen, während die subglandulären Partieen sehr stark mit ihnen besetzt sind. Fe-Cyten habe ich bei diesem Thiere im Coecum nicht durch das Epithel wandern sehen.

Oberer Dickdarm. Die Anzahl der Fe-Cyten ist hier bedeutend geringer. Sie sind zum Theil zerfallen und geben einen bräunlichen Farbenton bei Berlinerblau-Reaction. Das Epithel zeigt wenig Bilder von Auswanderung der zelligen Gebilde.

Unterer Dickdarm. Fast derselbe Befund wie im vorherigen Darmtheil.

Leber. Fe-Reaction findet sich hier in der Gegend des portalen Theiles der Läppehen und zwar an Leukocyten kleiner Form gebunden. Die Centralvenen sind stark mit Blut gefült, wie auch die zu ihnen hinziehenden Capillaren. In den Leberzellen beobachtet man die für Hungerzustände charakteristischen Vacuolen und zwar sind dieselben so angeordnet, dass man im centralen Theile der Läppchen Vacuolen von kleinem Kaliber begegnet, während in portalen dieselben zu grösseren zusammengetreten sind.

Milz. Die Reaction ist etwas schwächer als bei normalen Thieren, und zwar scheint es, dass die in der Nähe der Trabekeln im adenoiden Gewebe befindlichen Fe-haltigen Schollen, wahrscheinlich Reste untergegangener Erythrocyten, hier an Masse geringer sind. Sonst ist die Vertheilung der Fe-haltigen Leukocyten wie bei den normalen Thieren. Im Hilus angeschnittene Venen zeigen deutlich Fe-Cyten im Lumen.

\section{Meerschweinchen $\mathrm{A}$.}

Dieses um 3 Monate ältere Thier wurde an 11. Tage des Versuches matter. Nachdem es noch kurz vorher vom Brot genagt hatte, wurde es entblutet.

Section. Der Magen ist durch Gase aufgetrieben und mit nicht unbedeutendem consistenteren, gelblich bräunlichen Inbalte gefüllt. Viele kleine Klümpchen haften der Schleimhaut sehr fest an. Die eröffneten Darmtheile präsentiren eine äusserst blasse Mucosa. $\left(\mathrm{NH}_{4}\right)_{2} \mathrm{~S}-$ Behandlung der einzelnen Darmtheile gibt ganz denselben Befund wie beim vorhergehenden 'Thier.

Mikroskopisches. Magen: negativer Befund.

$\mathrm{D}$ u o d e n u m. Auch hier keine Spur von feinkörniger Einlagerung von Fe-haltigen Körnchen im Epithel. Die Anzahl der Fe-Cyten ist in diesem Falle nicht so bedeutend wie beim vorhergehenden Indi- 
viduum. Ausserdem erscheint die Intensität der Fe-Reaction an den Fe-Cyten geringer, sie sind nicht so intensiv blau bei Ferrocyankali $+\mathrm{HCl}=$ und $\left(\mathrm{NH}_{4}\right)_{2} \mathrm{~S}$-Behandlung, wie das im vorherigen Objecte der Fall war. Die Epithelzellen, besonders an den Spitzen der Zotten, weisen Vacuolen auf, die, von dem kleinsten Umfange beginnend, zu recht bedeutendem zusammenfliessen.

Il eum. Fe-Cyten grosser Form sind in ziemlich bedeutender Menge in dem Stroma der Zotten; besonders viel sieht man sie in den Capillaren, welche den Chyluscanal umgrenzen. Ausser den grossen Formen begegnet man auch kleineren Fe-Cyten, aber weniger oft. In den Epithelzellen, hauptsächlich des oberen Drittels der Zotte, sind sehr bedeutende Vacuolenbildungen zu constatiren. An den Spitzen vieler Zotten ist das Epithel abgestossen, und Leukocyten, wie Fe-Cyten gelangen in das Darmlumen. Auf letztere Erscheinung, die zu den Kunstproducten zu zählen ist, komme ich später zu sprechen.

Coecum. Hier ist die Fe-Reaction sehr stark, und zwar sind es die Fe-Cyten grosser Form im adenoiden Gewebe, die in den interglandulären Partieen die Lücken ausfüllen.

Sodann bemerkt man sehr stark ausgesprochene Trichterbildungen. Die langen Canäle, die trompetenartig zum Darmlumen hinziehen, sind an manchen Stellen Fe-haltig; es findet sich Fe-Reaction, gebunden, wie es scheint, an Flüssigkeiten, die über die Wände der Canäle hingegangen. Im Darmlumen sehr zahlreich Leukocyten kleiner Form (Wanderzellen) zu sehen. Dieselben liegen meist ganz in der Nähe des Epithels und sind sehr deutlich von den Zellkernen der Epithelien durch ihre viel stärkere Tinction mit Alaun-Carmin zu unterscheiden. In der Submucosa sind die Fe-Cyten hier und da anzutreffen, aber im Ganzen selten.

Rectum. In diesem Darmtheile findet man grosse Fe-Cyten in den Interstitien zwischen den Lieberkühn'schen Drüsen. Auswanderung direct nicht zu beobachten. In der Submucosa keine FeCyten vorzufinden.

Leber. In den peripheren Theilen der Leberläppchen findet man Fe-Reaction in diffuser Form in den Leberzellen.

In den Capillaren begegnet man ziemlich vielen grossen und kleinen Fe-Cyten, die langgestreckt sich dem Lumen der Capillaren anpassen. An den Leberzellen selbst nimmt man keine Vacuolenbildung wahr. Auffallend ist die starke Füllung der grösseren 
Ueber die Resorption und Ausscheidung des Eisens im Darmcanale etc. 483

Gallengänge. Das Epithel derselben ist einfach cylindrisch und weist keine entzündlichen Processe auf. Die Central-, wie auch die Portalvenen sind stark gefüllt. Die Kerne der Leberzellen sind gut durch Carmin gefärbt. Am Protoplasma einiger Leberzellen findet sich beginnende körnige Trübung.

Milz. Die Fe-haltigen Schollen sind noch spärlicher vorhanden, als das im vorigen Meerschweinchen der Fall war. Im Uebrigen entspricht das Bild ganz dem rorhergehenden Falle, auch hier sieht man in der angeschnittenen Vene deutliche Fe-Cyten grosser Form.

\section{Meerschweinchen absoluter Carenz ansgesetzt.}

1. Am 25. Januar 1897 wurde ein männliches Meerschweinchen von $345 \mathrm{~g}$ Gewicht unter die Glasglocke gesetzt und erhielt weder Futter, noch Wasser. Der Koth wurde mehrmals täglich entfernt und die Glasplatte, auf der die Glocke stand, täglich gewaschen. Nach $3 \times 24$ Stunden wurde es so schwach, dass es sich nicht mehr auf den Beinen halten konnte, und ohne noch den Tod abzuwarten, kam es zur Entblutung.

Section. Im Magen befinden sich mässige Mengen eines bräunlichen, festeren, mit Haaren vom Meerschweinchen untermischten Inhalts. Dünn- und unterer Dickdarm leer. Das Coecum und der obere Dickdarm sind mit bedeutendem, pfefferkuchenartigem Inhalt angefüllt.

In $\left(\mathrm{NH}_{4}\right)_{2} \mathrm{~S}$ wird das Duodenum sehr bald im Zottentheile dunkelschwarzgrün; Jeju num und Ile u m verfärben sich fast ebenso stark. Coecum und Dickdarm geben ebenfalls eine sehr kräftige Reaction. Das Thier hatte um $22 \%$ des Anfangsgewichts abgenommen.

2. 10. März 18:8. Ein weibliches Meerschweinchen von $492 \mathrm{~g}$ wird unter die Glasglocke gesetzt. Es lebte ohne Futter und Wasser $8 \times 24$ Stunden und nahm um $39 \%$ seines Anfangsgewichts ab. Es verhielt sich während der ganzen Hungerzeit vollkommen ruhig, sank dann am 8. Tage um und kam anscheinend todt zur Section. Bei der Fröffnung der Brusthöhle contrahirte sich noch das Herz. Bei der Eröffnung der Baurhhöhle sah man durch die Serosa durehscheinende dunkle Flecken am Magen. Nach Eröffiung derselben fanden sich braune, mit Blut untermischte Massen in ihm. Die Mucosa wies mehrere linsengrosse Blutextravasate auf. Dünndarm 
und Dickdarm leer, während das Coecum mässige Mengen braunen, flüssigen Inhaltes enthielt. Die Schleimhaut des Darmes vom Magen abwärts durchweg stark hyperämisch, ohne jedoch Ekchymosen aufzuweisen. $\left(\mathrm{NH}_{4}\right)_{2} \mathrm{~S}-\mathrm{B}$ ehandlung ruft am Magen eine geringe Grünfärbung, am Du odenum, Jejunum, Ileum eine ziemlich gleichmässige schwärzlichgrüne Reaction hervor. Coecum und oberer Dickdarm werden dunkler verfärbt.

Mikroskopisches. Der Magen giebt bei beiden Thieren negativen Befund.

Das Duodenum zeigt bei beiden Thieren keine Spur einer Fe-Reaction in feinkörniger Ablagerung im Epithel. Im Stroma fanden sich hingegen sehr schön ausgeprägte, starke Fe-Reaction zeigende Phagocyten. Sie haben einen meist excentrischen Kern und enthalten Einschlüsse, eckige Partikel von verschiedener Grösse und wechselndem Fe-Gehalt. Die Zahl der Fe-Cyten ist recht bedeutend. Bilder, die auf ein Durchwandern durch das Epithel schliessen lassen könnten, habe ich nicht gefunden, wenngleich ausserhalb im Darmlumen Fe-Cyten an der Cuticularmembran hafteten.

Das zweite Thier zeigte geringere Reaction und unterschied sich noch dadurch, dass die Epithelie» an der Zottenspitze Vacuolen aufwiesen.

Jejunum. Während das erste Thier eine geringere Reaction an den Fe-Cyten aufweist, und auch die Anzahl derselben geringer ist, findet das Umgekehrte beim zweiten statt. Die Fe-Cyten sind meistens von sehr grosser Form, ausserdem stark angefüllt mit heilen Erythrocyten und Trümmern derselben. Ein Solitärfollikel zeigt an der Serosaseite einige spärliche Reste Fe-Reaction zeigender Schollen. Der Blutreichthum ist in diesem Darmtheile des zweiten Thieres sehr gross, man sieht viele Trümmer der Erythrocyten im Stroma der Zotten selbst liegen.

Im Il eum derselbe Befund wie im Jejunum. Coecum. Das interglanduläre Gewebe ist stark angefüllt mit Fe-Cyten, die viel grösser sind als diejenigen in den beschriebenen Darmtheilen. Auch hier sind sie hauptsächlich mit Erythrocyten und deren Trümmern besetzt. Am Oberflächenepithel haben sich die trichterförmigen Bildungen etablirt, durch welche Erythrocyten und Fe-Cyten in's Darmlumen gelangen.

Leber. Keine Spur von Fe-Cyten zu bemerken. 
Milz. In der Pulpa sieht man grosse Schollen von der Menge wie bei Thier $B$ von nicht charakteristischer Vertheilung in der Pulpa. Man bekommt aber den Eindruck, dass die Schollen aus Erythrocyten hervorgegangen sind, die im Leibe der Phagocyten sich befunden haben; man sieht nämlich noch viele von den letzteren, angefüllt mit heilen Erythrocyten. Die Malpighi'schen Körperchen zeigen keine Reaction, ihre Capillaren sind aber stark gefüllt.

Fassen wir die Resultate der beiden Abschnitte zusammen, so finden wir, dass die Fe-Reaction im Duodenum, trotz Fe-armer Nahrung und Entziehung jeglichen Futters während acht Tagen, nicht geschwunden ist. Die feinkörnige Ablagerung in den Epithelien des Duodenum fehlte, aber in den Phagocyten konnte deutliche Reaction nachgewiesen werden. Dass die dargereichte Nahrung wirklich Fe-arm war, war durch die Abwesenheit der feinkörnigen Ablagerung im Epithel erwiesen. Der Koth war äusserst sorgfältig während des Tages entfernt worden und jede Berührung mit Fehaltigen Substanzen vermieden. Der Einwurf, es könnte die Galle die Ursache sein, lässt sich jetzt nicht von der Hand weisen. Daher versuchte ich die Unterbindung des Ductus choledochus.

\section{Untersuchung des Darmcanals nach Unterbindung des Ductus choledochus.}

Fünf Meerschweinchen, von denen eines weiblichen Geschlechtes war, wurden laparatomirt und der Ductus choledochus unterbunden. Die Operation, unter antiseptischen Cautelen ausgeführt, gelang bei dreien vollkommen; zwei gingen dureh Sepsis ein. Da die Technik der Operation leichter bei weniger gefülltem Darme vor sich geht, liess ich die Thiere $1-2 \times 24$ Stunden vor der Operation hungern. $A$ starb 17 Stunden, $B$ 6 Stunden nach der Operation an den Folgen der Sepsis. $C$ wurde $2 \times 24$ Stunden, $D 3 \times 24$ Stunden nach der Operation entblutet. $E$ wurde entblutet, nachdem es $1 \times 24$ Stunden ohne und $1 \times 24$ Stunden mit Maulkorb nach der Operation gehungert hatte. Bei der unausgesetzten Beobachtung der Thiere bemerkte ich, dass letztere sehr bald Fresslust zejgten und aus Mangel an Futter den ausgeschiedenen Koth, wenn er nicht schnell genug entfernt wurde, frassen. Daher stellte ich das Thier $D$ auf ein Drahtgitter aus Messing. Diese Maassnahme erwies sich aber auch nicht als ausreichend. Als ich nämlich das Thier $D$, das sich 
unter der Glasglocke auf dem Gitternetze befand, eine starke Beugung machen sah, glaubte ich, dass es an der Operationswunde nage. Es erwies sich aber, dass es den Koth mit den Zähnen ergriff, bevor er durch das Gitternetz fiel, also für das Thier unerreichbar wurde. Jetzt war es klar, warum die Thiere, die doch alle $1-2 \times 24$ Stunden vor und mehr weniger dieselbe Zeit nach der Operation gehungert hatten, einen stark gefüllten Magen aufwiesen. Daher stellte ich den Versuch mit Thier $E$ so an, dass ich es nach der Operation $1 \times 24$ Stunden ohne und $1 \times 24$ Stunden mit Maulkorb hungern liess.

Die operirten Thiere boten viel pathologisch-anatomisch Interessantes dar, das mit der Fe-Frage in keinem directen Zusammenhange steht.

Die Befunde der früh verstorbenen Thiere wichen in Betreff des Fe-Gehaltes insofern von den günstig operirten ab, als die feinkörnige Ablagerung im Duodenalepithel bei ihnen vorhanden war, bei den anderen aber, mit Ausnahme des Thieres $D$, fehlte. Die Unterbindung des Ductus choledochus war bei allen Thieren vollkommen gelungen, die Gallenblase erreichte bei einigen fast Taubeneigrösse. Der Mageninhalt verschwand erst bei Thier $E$, dem der Maulkorb aufgesetzt worden, vollkommen, während er bei allen übrigen ausnahmslos in bedeutender Menge vorhanden war.

$\left(\mathrm{NH}_{4}\right)_{2} \mathrm{~S}$-Behandlung des Magens ergab bei allen Thieren eine grünliche Färbung, wie bei allen vorher besprochenen normalen und Hungerthieren.

Das Duodenum wurde stark dunkelgrün, wie das Jejunum, verfärbt.

Das Il eum ergab eine schwächere Reaction.

Das Coecum wurde sehr stark schwarzgrün, ebenso der ob er e Dickdarm.

Der untere Dickdarm war schwächer verfärbt.

Da mir nur der letzte Fall, Meerschweinchen $E$, einwandsfrei in Bezug auf Erfüllung aller vorausgesetzten Bedingungen erscheint, so möchte ich das Protokoll desselben anführen. Die mikroskopische Untersuchung des Meerschweinchens $D$ hatte ergeben, dass im Duodenalepithel eine Resorption des Eisens in feinkörniger Ablagerung nachzuweisen war. Da das Thier $1 \times 24$ Stunden vor und $3 \times 24$ Stunden nach der Operation gehungert hatte, so konnte es nur der Koth gewesen sein, aus dem das Eisen stammte, denn die 
Ueber die Resorption und Ausscheidung des Eisens im Darmcanale etc. 487 vollkommene Verhinderung der Aufnahme desselben war nicht erreicht worden, was die Section dargethan hatte. Diesem Resorptionsvorgange entsprechend, waren unzweifelhafte $\mathrm{Fe}$-Reaction aufweisende Phagocyten im Duodenum sowohl, wie auch im Jejunum und Ileum vorgefunden worden. Um nun eine absolute Verhinderung des Kothfressens zu erzielen, liess ich einen Maulkorb aus Messingdraht verfertigen, der dem zu operirenden Thier gut passte. Nachdem das männliche Meerschweinchen von $370 \mathrm{~g}$ Gewicht zwei Tage der absoluten Carenz unterzogen worden, wurde es am 18. April 1897 laparatomirt und der Ductus choledochus unterbunden. Nach der Operation hungerte es 24 Stunden ohne Maulkorb und darauf mit dem Maulkorb abermals 24 Stunden. Da es jetzt so schwach war, dass es sich nicht mehr erheben konnte, schnell athmete und Zuckungen an den Extremitäten auftraten, wurde es durch Enitbluten getödtet.

Section. Die Bauchwunde sieht sehr gut aus. Nach Durchschneiden der fortlaufenden Naht lösen sich die Ränder der Wunde durch eine gelinde Gewaltanwendung von einander. Die Därme sind nirgends adhärent, in der Bauchhöhle keine Flüssigkeit. Der Magen ist ganz collabirt; bei Fröffnung desselben erweist es sich, dass er, ausser einer geringen Menge ganz klarer, durchsichtiger, stark sauer reagirender, schleimiger Flüssigkeit, nichts von festen Bestandtheilen enthält. Duodenum und Jejunum sind vollkommen leer, im Ileum befindet sich eine ganz geringe Menge weisslichen Inhaltes. Coecum und oberer Dickdarm enthalten braune Kothmassen. Der Ductus choledochus richtig unterbunden, die Gallenblase vom Umfange einer grossen Haselnuss.

$\left(\mathrm{NH}_{4}\right)_{2} \mathrm{~S}$ ruft makroskopisch im Magen eine hellgrüne Farbe hervor; das Duodenum zeigt eine Färbung wie bei normalen Thieren; im Jejunum und Il eum ist sie schwächer. Im Coecum und Dickdarm ist die Reaction stark. Im frischen Zupfpräparat sieht man unter dem Mikroskope deutliche Fe-Cyten im ganzen Dünndarme.

Mikroskopisches.

Magen gibt negativen Befund.

Duodenum. Das Epithel zeigt nirgends eine feinkörnige Ablagerung. Fe-Cyten hingegen findet man in grosser Menge in den Spitzen der Zotten, wie auch an manchen Zotten bis zur Mitte der- 
selben. Die Blutcapillaren sind stark mit ibnen angefüllt. An der Auswanderung betheiligen sich Fe-Cyten und Leukocyten. Im Darmlumen sieht man Epithelzellen, die sich vom Epithellager gelöst haben, Fe-cyten, Leukocyten und Erythrocyten. Dass man sich diese Elemente aber nicht alle als durch das intacte Epithellager hindurch gewandert vorzustellen hat, ersieht man daraus, dass an einigen Zotten das Epithel Risse aufweist, die künstlich entstanden sind. Durch das Hineinlegen der frischen Darmstücke in die Fixationsflüssigkeit (Formalinlösung) contrahirt sich die Zotte so stark, dass die aus dem Stroma gepresste Flüssigkeit die Epitheldecke abhebt und Risse in derselben verursacht. Dabei treten dann auch leicht die im Stroma enthaltenen Zellen in das Darmlumen über.

Ich spreche daher von Auswanderung nur da, wo ich deutliche Bilder derselben am unversehrten Epithel beobachtet habe.

Karyolytische Figuren sieht man an den Epithelzellen der Zottenspitzen, wie auch an den im Darmlumen befindlichen, in Schleim gebetteten Leukocyten.

Oft begegnet man Bildern, die an den Spitzen der Zotten eine Oeffnung darstellen, durch welche Zellen aus dem Stroma der Zotte in das Darmlumen gelangen. Solche Bildungen im Epithel sind von Grünhagen ${ }^{1}$ ) beschrieben und "Porus" genannt worden. In Berücksichtigung dessen, was ich oben von dem Schicksal der in Formalinlösung gebrachten frischen Darmstücke gesagt habe, muss ich Heidenhain ${ }^{2}$ ) beistimmen, wenn er genannte Bildungen als durch Risse im Epithel, während der Fixirung entstanden, ansieht.

Jejunum. Fe-Reaction hier ebenso stark wie im Duodenum. Im Leibe der Fe-Cyten grosser Form findet man Erythrocyten, die von ihrer normaler Weise gelblichgrünen Farbe alle Abstufungen bis zu der gesättigten berlinerblauen darbieten.

I le um. Im Allgemeinen der Befund wie im Duodenum. Diapedese der Erythrocyten hier oft zu beobachten.

Co ecum. Die interglanduläre Partie der Mucosa ist stark Fehaltig. Man erkennt die Fe-Cyten nicht deutlich in ihren Umrissen. Sie sind nämlich so eng an einander gepresst, dass man sie nicht

1) Grünhagen, Ueber Fettresorption und Darmepithel. Arch. f. mikrosk. Anat. Bd. 29.

2) Heidenhain, l. c. S. 5. 
von einander unterscheiden kann. In die in der Submucosa befindlichen Venen sieht man von den Interstitien des Bindegewebes her zahlreiche Fe-Cyten grosser Form gelangen. Sie bleiben in dem perivasculären Gewebe der Venen, in letztere hinein sieht man sie sich nicht begeben. Die Li eberkühn'schen Krypten erscheinen erfüllt mit einer klaren Flüssigkeit.

Der Fundus ist ballonartig erweitert, und die Drüsenzellen selbst sind plattgedrückt in Folge des im Lumen herrschenden Druckes. Aus dem Lumen der Drüsen sieht man hier und da ein aus geschrumpften Mucinfäden zusammengesetztes Gebilde hervorgehen. Leukocyten findet man oft in den Krypten, Fe-Cyten hingegen sah ich auch hier niemals.

Das Oberflächenepithel ist verhältnissmässig gut erhalten, zeigt keine Vacuolenbildurig. In den Interstitien der Epithelzellen trifft man hier und da lang gestreckte Erythrocyten auf dem Wege in das Darmlumen begriffen.

Oberer Dickdarm. Fe-Reaction wie im Coecum. Trichterförmige Canäle haben sich am Oberflächenepithel gebildet. Eine zähe, bräunlich gefärbte Masse ergiesst sich aus ihnen. Auch Erythrocyten sieht man diesen Weg nehmen.

Unterer Dickdarm. Fe-Reaction im interglandulären Theile viel schwächer als im oberen Dickdarm. In der subglandulären Partie, wie auch in dem Bindegewebe der Submucosa gar keine Fehaltige Zellen anzutreffen. Die trichterförmigen Canäle des Oberflächenepithels scheiden kenlenförmige Gebilde aus, von denen viele schon im Darmlumen sich befinden. Sie sind die Abgüsse einer zähflüssigen Masse in den Canälen. Die Farbe der sich ausscheidenden Substanz ist bräunlichgrün, und ist letztere aus einer Auflösung der in grossen Mengen im interglandulären Bindegewebe angesammelten Erythrocyten hervorgegangen. In Schüben wird diese Masse durch die trichterförmige Canäle hinausbefördert, wobei die gewissermaassen erstarrende Masse die Form der Canäle annimmt. Die Krypten betheiligen sich an diesem Vorgange gar nicht, man sieht in ihnen niemals Erythrocyten.

Leber. Fe-Reaction nur hier und da in einer Capillare, an Schollen gebunden, anzutreffen.

Milz. In der Pulpa sehr starke Fe-Reaction. Der Einschmelzungsprocess der Erythrocyten ist hier stärker als bei den 
Milzen der Abschnitte. II und III. Die Malpighi'schen Körper frei von Reaction.

Lymphknoten. Um den ganzen Mesenterialknoten herum sieht man hart unter der Kapsel grosse Fe-Cyten mit wechselndem Fe-Gehalte. Die Einschlüsse sind, ausser kleinen Leukocyten, hauptsächlich Erythrocyten, deren mehr oder weniger manifest gewordene Eisenmengen dann die entsprechend stärkere oder schwächere FeReaction bedingen.

Die Körnelung der Fe-haltigen Elemente in den Fe-Cyten hier ist feiner als in den Fe-Cyten der Milz.

Trotz Aussehlusses der Galle und des Kothfressens war die Fe-Reaction im Duodenum nicht ausgefallen. Die Annahme Macallum's, dass die beim Hunger von einer Woche noch fortbestehende Fe-Reaction von der Galle herrühre, konnte durch meine Versuche widerlegt werden. Die soeben beschriebenen Untersuchungen wiesen ausserdem darauf hin, dass der Koth, der von den Thieren so begierig aufgefargen wird, in Fällen, wo die gewohnte Nahrungszufubr ausbleibt, keine unbedeutende Rolle spielen muss. Um mir Klarheit über diese Verhältnisse $z u$ verschaffen, unternahm ich Versuche, die im folgenden Abschnitte beschrieben werden sollen.

\section{Befund im Darmcanale der Meerschweinchen bei absoluter Carenz mit Application eines Maulkorbes.}

Wie wir im vorigen Capitel sahen, war die Fe-Reaction trotz Ausschlusses der Galle und des Kothfressens nicht ausgeblieben. Die Epithelien zeigten keine Spur einer feinkörnigen Ablagerung, die Phagocyten hingegen wiesen eine unzweideutige Fe-Reaction auf. Der Grund für die noch vorhandene Fe-Reaction hätte nun liegen können:

1. darin, dass die Duodenalzotten noch nicht ganz frei von anhaftenden Fe-haltigen Koththeilchen geworden waren;

2. darin, dass der Magensaft selbst, der viel Fe-haltiger als das Gallensecret ist, die Reaction verursachte;

3. darin, dass die vorhergegangene Operation der Unterbindung des Ductus choledochus an sich Bedingungen geschaffen hat, die in einer uns unbekannten Weise eine Fe-Reaction in den Phagocyten ermöglicht.

Um nun zuerst Bedingungen zu schaffen, unter welchen die Duodenalzotten als absolut frei von anhaftenden Koththeilchen an- 
Ueber die Resorption und Ausscheidung des Eisens im Darmcanale etc. 491

zusehen wären, musste eine Versuchsreihe mit Anwendung des Maulkorbes angestellt werden. -

Einem normalen Meerschweinchen sollte ein Maulkorb aufgesetzt und das Thier nach 24 Stunden getödtet werden.

Falls bei der Section im Magen sich Inhalt erwiesen hätte, sollte mit neuen Versuchen so lange fortgefahren werden, bis ein Stadium des Hungers erreicht worden wäre, wo Magen und Duodenum sich vollkommen frei von Inhalt präsentirt hätten.

Wenn jetzt bei der mikroskopischen Untersuchung sich gezeigt hätte, dass die Fe-Reaction in den Phagocyten vorhanden war, so konnte geschlossen werden, dass diese Reaction in den Phagocyten des Duodenums der Meerschweinchen, die eine Woche ohne Maulkorb gehungert hatten, durch den verzehrten Koth verursacht war.

Die Application eines Maulkorbes ist beim Meerschweinchen mit nicht geringen Schwierigkeiten verbunden, wenn er gut passen soll, ohne unerwünschte Erseheinungen herbeizuführen. Fin allzu schonungsvoll aufgesetzter Maulkorb wird bei den ersten Bestrebungen, sich davon zu befreien, vom Versuchsthier abgerissen; bei festerem Anziehen des um den Hals gehenden Drahttheiles des Apparates bemerkt man sehr bald Oedeme der linken oder rechten Ohrmuschel. Bei einem Meerschweinchen musste ich die Vorderbeine fesseln, um ihm die Möglichkeit zu nehmen, sich vom Maulkorbe zu befreien. Wenn nun endlich der aus Messingdraht den Hundemaulkörben nachgebildete Apparat dem Thiere zweckentsprechend angelegt worden, so führt letzteres die verzweifeltsten Anstrengungen aus, um sich von der ungewohnten Vorrichtung zu befreien. Indem die Thiere mit den Vorder- und Hinterfüssen in das Halsband zu gelangen suchen, führen sie Sprünge aus, bei denen sie sich nach hinten überschlagen. Aehnliches konnte ich am Kaninchen ${ }^{1}$ ), das in gleicher Weise behandelt worden, beobachten. Nach einigen Stunden beruhigten sich die Thiere, ergeben in ihr Schicksal.

Die von mir benutzten Versuchsthiere bekamen hauptsächlich Gras $^{2}$ ) zur Nahrung, Brot wenig; ich hoffte, hierdurch mir die Unterscheidung frischen Mageninhaltes von verzehrtem Kothe zu erleichtern.

1) G. Swirski, Zur Frage über die Retention des festen Mageninhaltes beim hungernden Kaninchen. Arch. f. exper. Pathol. u. Pharm. Bd. 41 S. 143.

2) Die Versuche in diesem Abschnitte sind vom Fnde des April bis zur Mitte des Mai 1897 angestellt worden. 
Der Hunger mit Maulkorb wird von den Thieren unbedingt schlechter vertragen als derjenige ohne Maulkorb. Das Meerschweinchen $D$ hätte, allem Anschein nach, nicht länger als $4 \times 24$ Stunden gelebt, da das Stadium der Lähmung schon eingetreten war. Will man den Fall als nicht beweisend ansehen wegen des geringen Gewichtes des Thieres (350 g), so kann auf das Thier $E$ verwiesen werden, das $629 \mathrm{~g}$ schwer, nach Verlauf von $5 \times 24$ Stunden genau in demselben Zustande sich befand wie $D$. Die Thiere wurden täglich gewogen. Die Gewichtsverluste verhielten sich folgendermaassen :

\begin{tabular}{|c|c|c|c|c|c|c|c|}
\hline 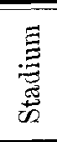 & 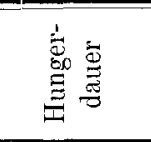 & 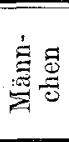 & 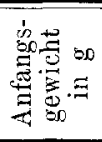 & 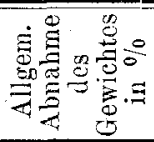 & $\stackrel{\overrightarrow{0}}{2}$ & 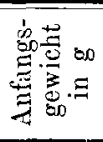 & 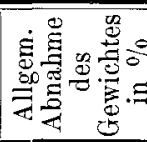 \\
\hline $\begin{array}{l}\text { I. } \\
\text { II. } \\
\text { III. } \\
\text { IV. } \\
\text { V. }\end{array}$ & $\begin{array}{l}1 \times 24 \quad \text { St. } \\
2 \times 24 \quad " \\
3 \times 24 \quad " \\
4 \times 24 \\
5 \times 24 "\end{array}$ & $\begin{array}{l}A \\
B \\
C \\
D \\
E\end{array}$ & $\begin{array}{l}511 \\
462 \\
470 \\
350 \\
629\end{array}$ & $\begin{array}{l}20,3 \\
24,4 \\
32,5 \\
25,4 \\
26,8\end{array}$ & $\frac{A}{C}$ & $\begin{array}{l}\frac{440}{6} \\
- \\
-\end{array}$ & $\begin{array}{c}\frac{13,5}{19,6} \\
- \\
-\end{array}$ \\
\hline
\end{tabular}

Bei den vorgenommenen Sectionen liess sich constatiren, dass der Mageninbalt gradatim abnahm, und zwar fand ich den Magen bei Thieren, die $3 \times 24$ Stunden mit Maulkorb gehungert hatten, vollkommen leer. Beim Hunger von $2 \times 24$ Stunden fand ich noch deutliche Grasfasern vor. Der Magen enthielt, falls er vollkommen frei von Inhalt war, was nach $3 \times 24$ Stunden Hunger mit Maulkorb stets der Fall war, eine klare, schleimige Flüssigkeit, die sauer und nur in einem Falle $(C)$ alkalisch reagirte.

Das Duodenum war vom vierten Hungertage ab frei von jeglichem festeren Inhalte.

Das J ejunum enthielt meistens Flüssigkeit.

Im Il e um war in zwei Fällen festerer Inhalt gefunden worden.

Im Coecum waren stets wechselnde Mengen braunen Kothes. Die geringste Füllung des Coecums wies das Thier $D$, nach einem Hunger von $4 \times 24$ Stunden, auf. Bei demselben Thier waren die Dünndärme fast absolut frei von consistenterem Inhalt, während im oberen Dickdarme eine geringe Menge sich vorfand und das Rectum vollkommen frei davon war. -

Die Mucosa bot makroskopisch eine mit den Hungertagen zunehmende Hyperämie dar, die schliesslich im V. Stadium zu sehr bedeutenden Ekchymosen in der Magenschleimhaut sich steigerte. 
Die Ekchymosen waren schon von aussen an dem stark mit Gasen gefüllten Magen als schwarze Flecke zu erkennen, über welche das glänzende Peritoneum hinwegging. In diesem Stadium war das Duodenum mit klarem Schleime gefüllt, während im Jejunum und Ileum Schleim sich vorfand, dem Blut beigemengt war.

$\left(\mathrm{NH}_{4}\right)_{2} \mathrm{~S}$. - Behandlung. Der Magen nimmt in allen Stadien eine gleichmässig grüne Farbe an.

Das Duodenum der Thiere, die $1 \times 24$ Stunden gehungert haben, ist vou dem normalen nur wenig verschieden. Erst vom zweiten Tage ab lässt sich eine Verminderung der dunkelgrünen Färbung, die so auffallend vom Magen absticht, constatiren. Zum fünften Tage hin ist eine gradatim zunehmende Abschwächung der Reaction nachweisbar. Das Duodenum des Thieres $E$ zeigt eine braune bis braungrane Reaction; es fehlt das Grüne, das in den früheren Hungerstadien mit Maulkorb constatirt werden konnte.

Der Grund für diese Farbenänderung liegt in dem Mangel der locker gebundenen Fe-Partikel im Stroma der Zotten; es dringt der durch den starken Blutgehalt der Zotten bedingte braune Ton durch.

Jejunum und Ileum verhalten sich ebenso. Das Coecum gibt einen sehr geringen grünlichen Ton, der obere Dickdarm ebenfalls. Nur im unteren Dickdarm ist starke, grünschwarze Reaction, wie bei normalen Thieren, vorhanden.

$$
\text { Mikroskopisches. }
$$

Der Magen zeigt in keinem Stadium Fe-Reaction.

Stadium I. Im Duodenum fehlt die feinkörnige Ablagerung in den Epithelzellen vollkommen und tritt auch in keinem weiteren Stadium mehr auf, wesshalb ich ihrer daselbst nicht weiter erwähnen werde. Fe-Cyten sind schon etwas spärlicher als im normalen Thiere vertreten, zeigen aber kräftige Fe-Reaction. Man bemerkt dagegen viel Fe-haltige Schollen im Stroma der Zotten, was für einen Untergang von Phagocyten, die mit Exythrocyten geladen gewesen, spricht.

Jejunum. Die Fe-Cyten zeigen hier ein blasseres Aussehen als im Duodenum.

Il eum. Derselbe Befund wie im Jejunum. Das Epithel zeigt Vacuolen.

Stadium II. D u od en um. Fe-haltige Schollen wie im Stadium I. Fe-Cyten wenig vorhanden. Stärkere Blutfüllung des Stromas. 
Jejunum. Weder Fe-Cyten, noch Schollen zu sehen.

Ileum. In einigen Zotten Phagucyten mit sehr schwacher FeReaction. Vacuolen im Epithel.

Stadium III. D u od en um. Fe-Cyten von grossen Dimensionen mit kräftiger Reaction, aber nur in einigen Zotten anzutreffen. Man sieht die Fe-Cyten sehr stark mit Erythrocyten und Trümmern derselben gefüllt.

J ej un um. Vollkommenes Fehlen der Fe-Cyten.

Il eum. Wie das Duodenum. Hyperämie stark.

Stadium IV. Duodenum. Keine Fe-haltigen Schollen zu sehen. Die Fe-Cyten sind in ganz geringer Anzahl vorhanden.

J eju n u m und Il e um zeigen überhaupt keine Phagocyten mehr.

Stadium V. Duodenum. In den Zotten, die durch den Schwund des Stromas überhaupt ganz atrophisch geworden sind, keine Leukocyten zu sehen. Von Fe-Reaction absolut keine Spur mehr zu finden.

Jejunum und Ileum verhalten sich durchaus ebenso. Die Hyperämie ist durchweg colossal.

Coecum. Das interglanduläre Gewebe ist im Stadium I, fast wie bei normalen Thieren, mit Fe-Cyten gefült. Auch im Stadium II finden sich dieselben noch reichlich vor und geben eine gesättigte Fe-Reaction. Mit Stadium III wird die Reaction schon schwächer, während die Blutfüllung im Stroma des interglandulären Gewebes sehr zunimmt.

Im Stadium V sind die Phagocyten ganz hellgrün durch eingelagerte Trümmer von Erythrocyten; eine gesättigte BerlinerblauFärbung ist nirgends nachzuweisen. Es ist aber auch bier die Anzahl der Phagocyten selbst eine geringere geworden.

Oberer Dickdarm. Mit den Hungertagen nimmt die Anzahl der Phagocyten ab. Stadium $V$ weist weniger Phagocyten in diesem Darmtheile auf als im Coecum. Diapedese der Erythrocyten durch die Epithelien sehr deutlich zu beobachten.

Unterer Dickdarm. In den früheren Hungerstadien liessen sich Fe-Cyten im interglandulären Gewebe nachweisen. Im Stadium V aber sind solche nicht mehr zu finden.

Fe-Reaction sehen wir aber in den Capillaren des adenoiden Gewebes und zwar in diffuser Form.

Leber. Fe-Reaction begegnen wir in allen Stadien nur hier und da in wenig charakteristischer Weise in den Capillaren der 
Ueber die Resorption und Ausscheidung des Eisens im Darmcanale etc. 495

Läppchen, entweder an Leukocyten gebunden oder in Form feiner Körnchen.

Milz. In allen Stadien, besonders aber in den späteren, finden wir eine sehr starke Fe-Reaction in der Pulpa vor, und zwar ganz besonders in der Schollenform. Die Malpighi'schen Körper zeigen nur im Stadium $\mathrm{V}$ in der Nähe der Keimcentren grosse Fe-Cyten.

Lymphknoten verhalten sich ähnlich wie die Milz. Wenn in den ersten Stadien des Hungers hauptsächlich in den Lymphsinus Fe-Reaction, gebunden an Schollen und grosse Leukocyten, zu finden ist, sieht man in dem letzten Stadium auch in den Follikeln selbst Fe-Reaction zeigende Zellen. Die Einschmelzung der Erythrocyten geht in den späteren Hungerstadien, wie es scheint, in grösserem Maassstabe vor sich.

Das Resultat der Hungerversuche an Meerschweinchen mit Maulkorb ist, dass die Fe-Reaction, gebunden an die Epithelien des Duodenums, schon nach 24 Stunden schwindet. Die Reaction, gebunden an Phagocyten, besteht in abnehmendem Maasse bis zum vicrten Tage fort, wobei die Anzahl der Fe-tragenden Zellen selbst geringer wird. Bei dem Hunger von $5 \times 24$ Stunden konnten wir überhaupt keine Phagocyten im Dünndarme mehr finden. Im Coecum und oberen Dickdarme waren sie, wenn auch in verminderter Zahl, vorhanden. Im Magen war der Spcisebrei nach $3 \times 24$ Stunden nicht mehr nachzuweisen. Was daruach im Magen von festem Inbalt sich findet, kann daher nur a uf Rechnung des verzehrten Kothes kommen. Da dieser aber bei unseren Versuchen fortfällt, so muss auch in diesem Umstande der Grund für das Verschwinden der Phagoeyten im Dünndarme gesucht werden. Es fehlt das Irritament, das durch die Gegenwart des Kothes geboten wird. Diese Beobachtung erinnert an die Verhältnisse, die Heidenhain ${ }^{1}$ ) bci Hunden beschrieben hat. IIatten Iunde 4-7 Tage gehungert, so wiesen die Därmo eine geringere Anfüllung des adenoiden Gewebes mit Leukocyten auf als regelmässig ernährte Thiere. Fs sehwanden besonders rothkörnige Zellen (Fixation in Sublimat, Färbung mit $\mathrm{Eh} \mathrm{r-}$ li ch - B i o n d i'scher Flüssigkeit), die bei reichlicher Nahrungsaufnahme stark vertreten waren. Ehrlich ${ }^{2}$ ) selbst hielt diese Zellen nicht mit Sicherheit für eosinophile. Heidenhain kam bei seinen Unter-

1) Heidenhain, l. c. S. 76 .

2) Heidenhain, 1. c. S. 80.

E. Pflüger, Archiv für Plysigiogie. Bda 7 t. 
suchungen zu dem Schlusse, dass die genannten rothkörnigen Zellen auftreten, sobald nur ein mechanischer Reiz auf der Darmschleimhaut hervorgehracht wird. Die Art der Nahrung hat keinen Einfluss auf die Zellen. Die bei Hunger mit Maulkorb schwindenden Phagocyten des Meerschweinchens entsprechen, dem äusseren Verhalten nach, vollkommen den von Heidenhain beobachteten und auf Tafel IV, Fig. 28 u. 29, abgebildeten rothkörnigen Zellen ${ }^{1}$ ). Man hat nur die rothen durch die bei der Berlinerblau-Reaction grünblau erscheinenden Körnchen ersetzt zu denken.

\section{Meerschweinchen mit $\mathrm{Fe}$ gefiittert.}

Um die Verhältnisse im Darmcanale der Meerschweinchen bei Fütterung mit Fe-Präparaten zu studiren, verabfolgte ich einem Thiere Liquor ferri sesquichlorati, dreien Ferrum oxyd. sacchar. solubile (Schering).

Bei diesen Versuchen kam es mir hauptsächlich darauf an, die Wege der mikrochemisch nachweisbaren Aufnahme und Ausscheidung im Darmeanale zu untersuchen. Der Grund, wesshalb ich gerade die anorganischen Präparate dazu wählte, lag darin, dass ich zugleich für die Frage nach der Resorbirbarkeit derselben etwas beitragen zu können hoffte.

1. $0,5 \mathrm{~g}$ des officinellen Liquor ferri sesquichl. wurden mit $100 \mathrm{ccm}$ dest. $\mathrm{H}_{2} \mathrm{O}$ verdünnt, und mit dieser 0,5 procentigen Lösung getränktes Schwarzbrot wurde einem weiblichen Meerschweinchen $A$ von $480 \mathrm{~g}$ Gewicht vorgesetzt. Ausserdem erhielt es noch Kartoffeln und Kohlblätter. Im Laufe von 11 Tagen bekam es 94,5 mg met. Fe. Nachdem es noch zum letzten Male vom Brote gefressen, wird es eine halbe Stunde darauf chloroformirt und die einzelnen Theile bei noch schlagendem Herzen dem Körper entnommen. Dies geschah, um die herausgenommenen Darmtheile sofort von ihrem Inhalte in dest. $\mathrm{H}_{2} \mathrm{O}$ säubern und schnell in die Fixationsflüssigkeit bringen zu können.

2. Die mit Schering'schem Ferr. oxyd. sacchar. sol. gefütterten Thiere erhielten das Präparat in der Weise, dass $0,5 \mathrm{~g}$ davon in $50 \mathrm{ccm} \mathrm{H}_{2} \mathrm{O}$ gelöst und Schwarzbrotstücke damit getränkt wurden. Das Thier $B, 325 \mathrm{~g}$ sehwer, erhielt in 20 Tagen $75 \mathrm{mg}$ met. Fe und

1) Heidenhain, 1. c. S. 103. 
Ueber die Resorption und Ausscheidung des Eisens im Darmcanale etc. 497 wurde, bevor es zur Entblutung kam, mit einem Maulkorbe für $1 \times 24$ Stunden versehen.

Das Thier $C, 385 \mathrm{~g}$ schwer, erhielt während 15 Tagen $180 \mathrm{mg}$ Fe und wurde gleich nach der Mahlzeit getödtet.

Das Thier $D, 661 \mathrm{~g}$ schwer, verbrauchte in 4 Tagen $45 \mathrm{mg}$ Fe und wurde ebenfalls sofort nach der Mahlzeit getödtet.

Alle Thiere sind anscheinend bei der besten Gesundheit getödtet worden. Sie hatten während der Fütterungszeit zugenommen, bis auf das Thier $D$, welches um $50 \mathrm{~g}$ in 4 Tagen abnahm. Es ist das wohl nur dadurch zu erklären, dass es aus sehr guten Verhältnissen in die Einzelhaft kam und nicht Zeit gehabt hatte, sich an die neuen Verhältnisse zu gewöhnen.

Bei $\left(\mathrm{NH}_{4}\right)_{9} \mathrm{~S}$-Behandlung wird der Magen bei allen Thieren grünlich verfärbt, wie sonst unter normalen Verhältnissen.

Das Duodenum wird in allen Fällen kohlschwarz.

Das Jejunum färbt sich schwächer und

das 1 leum noch weniger stark.

Das Coecum wird kohlschwarz,

der obere Dickdarm weniger stark,

der untere Dickdarm wechselnd, bald wie der obere oder noch stärker verfärbt.

Leber und Milz werden kohlschwarz.

Die Leber des Thieres $D$ verfärbt sich nur wenig.

Die Niere wird in der Corticalsubstanz dunkler.

Die Marksubstanz der Mesenterialknoten wird meistens ganz schwarz, die Rindenpartie bleibt heller.

Die Peyer'schen Plaques werden durchweg dunkelgrün bis schwarz.

\section{Mikroskopisches.}

An der Magensehleimhaut liess sich in keinem Falle auch nur die geringste Fe-Reaction nachweisen.

D u od en um. Das Fpithel zeigte in allen Fällen die feinkörnige Ablagerung, wie wir sie bei den normalen Thieren fanden, nur in kräftigerer Reaction und einer grösseren Ausdehnung; indem sie sich auf viel mehr Zotten, als das bei normalen Thieren vorkommt, erstreckte. Ausserdem war noch eine diffuse Färbung der Epithelzellen zu constatiren, sowohl mit $\left(\mathrm{NH}_{4}\right)_{2} \mathrm{~S}$, wie auch bei der Berlinerblau-Reaction. Nur das Thier $B$, das vor dem Entbluten $1 \times 24$ St. 
mit Maulkorb gehungert hatte, zeigte keine Spur von feinkörniger Ablagerung, wie auch keine diffuse Reaction in den Epithelzellen. Die Fe-Cyten grosser Form sind sehr stark vertreten und füllen das Stroma der Zottenspitze stark an. Man kann sie von da aus in den Blutcapillaren bis in die Nähe der Basis der Zotten verfolgen. In dem centralen Chylusraum habe ich nie einen Fe-Cyten sehen können.

Die Reaction der Fe-Cyten ist entschieden gesättigter, als bei den normalen Thieren. Beim Thiere $A$, bei dem der centrale Chyluscanal etwas eröffnet war, fand ich eine diffuse Fe-Reaction ohne zellige Einlagerung. Eine leichte Hyperämie des Stromas war in einigen Präparaten nachweisbar; die Epithelzellen selbst wiesen vichts Abnormes auf.

Jejunum. An keinem Versuchsthier war eine feinkörnige Ablagerung in den Epithelzellen zu finden, wohl aber waren sie diffus verfärbt, mit Ausnahme von $B$.

Fe-Cyten grosser Form auch hier gesättigter, als normaler Weise. Das Thier $D$, das nur 4 Tage hindurch Fe erhalten hatte, bot in diesem Darmtheile an den Epithelien gar keine Reaction dar und in den Fe-Cyten eine ganz geringfügige. Gerade an den mit Fe gefütterten Meerschweinchen lässt sich die Zunahme der zelligen Elemente des adenoiden Gewebes der Schleimbaut im ganzen Darmtractus beobachten.

Man bemerkt hierbei die verschiedensten Eutwickelungsstufen: ganz junge Leukocyten nit rothem, wandständigem Kerne und rundem prallem Protoplasma mit noch sehr geringer Einlagerung von Fehaltigen Partikeln, dann wieder grössere mit stärkerer Einlagerung und schliesslich colossale Formen im Coecum und Dickdarm mit Einlagerungen von anderen zelligen Gebilden und stark Fe-haltigen Partikeln. Ich muss Heidenhain ${ }^{1}$ ) durchaus beistimmen, wenn er eine Bildung der Phagocyten im Stroma der Zotten selbst annimmt.

Die Durchsicht meiner Präparate führt mich zu derselben Anschauung.

Ein für die Resorptionsvorgänge in den Darmzotten interessantes Bild bietet das Jejunum des Thieres $C$. Auf einem feinen Schnitte kann man sich davon überzeugen, dass die Epithelzellen mit ihrem schmalen Theile, dem Fussende, sich an die hart unter dem Epithellager hinziehenden Blutcapillaren ansetzen. Durch

1) 1. c. S. 42 . 
Ueber die Resorption und Ausscheiding des Eisens im Darmcanale etc. 499

das Hineinlegen der frischen Darmstücke in die fixirende Flüssigkeit contrahirt sich manchmal die Zotte so sehr, dass aus dem Stroma derselben die darin enthaltene Flüssigkeit ausgepresst wird. Dadurch entstehen Ablösungen des Epithels vom Stroma. Heidenhain ${ }^{1}$ ) nimmt an, dass alle Fäden, die zwischen Epithelzellen und Stroma hierbei sich bilden, Artefacte seien.

Diesem kann ich nicht beistimmen. Sowohl an nur unvollständig abgelösten Epithelzellen, wie auch an günstig cefallenen, sehr feinen Sehnitten konnte ich einen deutlichen geweblichen Zusammenhang der Fpithelzellen mit don Blutapillaren nachwcisen. Für einen Theil der Epithelzellon wenigstens ist ein solcher Zusammenhang zweifellos. Der centrale Chylusraum ist an sehr vielen Zotten eröffnet und wird zu beiren Seiten von den Bluteapillaren eingefasst, in denen man ganze Reihen von grossen und kleinen Fe-Cyten, unterbrochen von Erythrocyten, sehen kann. Das Plasma selbst ist an manchen Stellen der Geftase Fe-haltig.

Der centrale Chylusram aber ist absolut frei von zelligen Flementen. Es findet sich in ihm nur die granulirte erstarrte Chylusflüssigkeit, die als solche ohne Weiteres von der Einbettungsmasse (Celloidin), die viel foiner granulirt ist, zu unterscheiden ist. An einigen wenigen Zotten ist diese Chylusmasse schwach Fe-haltig ${ }^{2}$ ).

I le um. Diffuse Fe-Reaction lässt sich auch hier in den Epithelzellen nachweisen, aber wiedor mit Ausnahme von B. - Fe-Cyten sind reichlich vorhanden; man sieht hier ebenfalls eine Proliferation derselben.

Wie im Jejunum findet man beim Thiere $C$ Zotten, deren centraler Chyluscanal erweitert und angefült ist mit der grobkörnigen, von der Einbettungsmasse verschiedenen Inbaltsmasse. Die $\mathrm{Fe}$ Reaction der Chylusflüssigkeit ist, ganz wie im Jejunum, nur an einigen Zotten $z \mathrm{u}$ constatiren. Wir finden hier öfter Bilder, wo die Zotte stark contrahirt ist und im Begriff steht, ihren Inlsalt weiter zu befördern. Das Epithel ist hier nirgends vom Stroma abgelöst. Wo das andeutungsweise vorhanden ist, überzeugt man sich von dem geweblichen Zusammenhange zwisehen Epithelzellen und Bluteapillaren.

1) 1. c. S. 21 .

2) cf. Tafel Vi Fig. 3. 
Wir haben in den soeben beschriebenen Jejunum- und Ileumzotten Bilder von Resorptionsvorgängen in diesen Darmtheilen vor uns. Wenn es zuzugeben ist, dass den Impuls zu der Contraction die Fixationsflüssigkeit gegeben hat, so ist es andererseits nicht von der Hand zu weisen, dass die Zotte, bevor sie in die fixirende Flüssigkeit gerieth, stark gefüllt war. Sie war in ihrer Thätigkeit begriffen, wo das Thier entblutet wurde und konnte den Act der Entleerung nicht zu Ende bringen. Durch den Reiz der Fixationsflüssigkeit contrahirte sich die Zotte, fand aber in den schon verengten Lymphräumen einen Widerstand für die Entleerung und erstarrte in dieser Situation. Diesen Resorptionsvorgang habe ich nur bei dem Thiere $C$ beobachten können.

Coecum. Die Zellen des Oberflächenepithels geben nur bei Thier $A$ Fe-Reaction, und zwar in diffuser Weise. Wir finden die Reaction in den Epithelzellen, die uns schon im Coecum des normalen Thieres II aufgefallen war. Die Intercellularsubstanz ist hierbei ebenfalls Fe-haltig, und man erhält den Eindruck, als ob eine Strömung von Fe-haltiger Flüssigkeit vom Darmeanale her durch die Intercellularsubstanz zum adenoiden Gewebe der interglandulären Partie hinzöge. Die Phagocyten, die an Zahl zugenommen haben, sind stark Fe-haltig. An den übrigen Versuchsthieren ist das Oberflächenepithel vollkommen frei von jeglicher Fe-Reaction. Die Zellen der Lieberkühn'schen Drüsen zeigen keine Reaction.

Das Coecum des Thieres $\mathrm{C}$ erinnert an das Bild, das ich bei der Besprechung desselben Darmtheiles des normalen Thieres geschildert habe. Aus dem Lumen der Lieberkühn'schen Krypten ergiesst sich eine Fe-haltige Flüssigkeit. Nur an einigen Drüsen beobachtet man zusammenhängende Stücke, die dann Pfröpfe bilden. Auch hier ist es der zum Darmlumen gerichtete Theil, welcher eine auffallend starke Fe-Reaction zeigt, während das zum Fundus gewandte Fnde sehr schwach verfärbt ist. In allen Fällen umgibt der Cuticularsaum der Drüsenzellen die Pfröpfe. Eine Einwanderung von Fe-Cyten durch die Drüsenzellen oder zwischen denselben hindurch ist mir hier ebensowenig zu beobachten gelungen, wie bei den übrigen.

Oberer Dickdarm. Die interglandulären Partieen sind mässig angefüllt mit Fe-Cyten. Am Oberflächenepitbel, welches diffuse Fe-Reaction zeigt, haben sich trichterförmige Canäle gebildet, durch welche Fe-Cyten kleiner Form in das Darmlumen gelangen. Die Wände der Canäle zeigen starke Fe-Reaction. Man erhält den 
Eindruck, als wenn von den Gebilden, die den Canal passirten, Fehaltige Theile an den Wänden zurückgeblieben wären.

Stöhr ${ }^{1}$ ) hat solche "Strassen", wie er sie nennt, durch welche die Leukocyten in das Darmlumen gelangen, in den Lymphknötchen des Darmes der Katze unter normalen Verhältnissen beobachtet.

Unterer Dickdarm. Meerschweinchen $C$ weist in diesem Darmtheile ein unzweideutiges Bild der Ausscheidung des $\mathrm{Fe}$ auf. In langen Streifen sieht man in den Blutgefässen des adenoiden Gewebes der interglandulären Partie der Mucosa $\mathrm{Fe}$-Reaction zeigende flüssige Massen, möglicher Weise zerfallene Fo-Cyten, zum Oberflächenepithel hinziehen und durch die trichterförmigen Canäle in das Darmlumen sich ergiessen.

Dass die Richtung unzweifelhaft zum letzteren hingeht, davon überzeugt man sich auf Schnitten, in denen noch Fäces an den Epithelzellen haften. In den Fäces sieht man dann Theile, von deren Herkunft aus den. Canälen man sich sofort überzeugen kann.

Aus den Lieberkühn'sehen Krypten sieht man keinen Fe-haltigen Inhalt hervorgehen. Die Schleimzellen sind colossal dilatirt und in überaus zahlreicher Menge vorhanden. - Nur auf Querschnitten kann man sich vom Vorhandensein der Protoplasmazellen überzeugen.

Leber. Fe-Reaction ist in den Leberzellen in diffuser Form bei allen Thieren zu finden, und zwar ist sie im peripheren Theil der Läppehen stärker als im centralen.

Am auffallendsten ist diese Anordnung der Reaction beim Thier B, das in 20 Tagen $75 \mathrm{mg}$ Fe verbrauchte und vor dem Entbluten einen Tag mit Maulkorb gehungert hatte.

Die instructivsten Bilder geben die Schnitte, wenn sie mit $\left(\mathrm{NH}_{4}\right)_{2} \mathrm{~S}$ behandelt sind. Es hebt sich nämlich der gelblich gefärbte centrale Theil der Läppehen mit dem Capillarnetze sehr effectvoll von dem dunklen, gesättigt grünen, portalen Theile der Läppchen ab. Die Leberzellen, unmittelbar um die Vena centralis herum, sind frei von $\mathrm{Fe}$; je mehr man sich der Peripherie der Läppchen nähert, um so stärker wird die Fe-Reaction in den Leberzellen. An manchen Läppchen nimmt man schon auf der Mitte des Weges zwisehen Centrum und Peripherie zuerst eine diffuse Fe-Reaction, dann auch allmählich eine Fe-haltige Körnelung in den Leberzellen

1) Stöhr, Ueber die Lymphknötchen des Darmes. Archiv für mikrosk. Anat. Bd. 33 S. 265. 
wahr, die in der Nähe der Portalgefässe selbst schon recht bedeutende Körner im Protoplasma aufweist. Der Kern der Leberzellen gibt keine Reaction. In den Capillaren verschwindend wenig Fe-Cyten zu bemerken. Die Lumina der angeschnittenen Portalvene zeigen überall eine deutliche Fe-Reaction in diffuser Form.

Milz. Bei den längere Zeit mit Fe gefütterten Thieren lässt sich ein stärkerer Fe-Gehalt constatiren, während die Milz des Thieres $D$, das nur 4 Tage hindurch $F$ e erhalten hatte, sich in keiner Hinsicht von der eines normalen Thieres unterschied. Bei den ersteren erscheinen die venösen Capillaren und Venen schärfer begrenzt von einem Rande, der allem Anscheine nach durch eine Membran gebildet wird, durch welche Fe-haltige Flüssigkeit dringt. Letztere wird von den in den Gefässen befindlichen Leukocyten aufgenommen, wobei sich constatiren lässt, dass gerade die der Membran am nächsten befindlichen Zellen die stärkste Reartion zeigen.

Im Pulpagewebe, das von den Capillaren begrenzt wird, sieht man sehr viele Fe-haltige Schollen und Fe-Cyten; diffuse Fe-Reaction ist aber nicht zu bemerken. Letztere beginnt erst am Rande des Gefässes, also schon innerbalb der venösen Capillaren und Venen. Im Hilus angeschnittene Venen zeigen bedeutende Mengen von FeCyten grosser und kleiner Form, während in den Arterien keine Fe-Reaction nachzuweisen ist.

Lymphknoten. In diesen fand ich keine besonders starke Reaction; sie unterschieden sich nicht so auffallend von den normalen Organen, wie es in der Milz der Fall war.

Wir sehen, dass durch Fütterung mit Liquor ferr. sesquichl. und Ferr. oxyd. sacehar. solub. bei Meerschweinchen eine unverkennbar stärkere Fe-Reaction im Darmeanale auftritt, als das bei normalen Thieren der Fall ist. Diese Reaction präsentirt sich in einer intensiveren und extensiveren Ablagerung von feinen, Fe-Reaction zeigenden Partikeln, wie auch in diffuser Form. Die erstere ist und bleibt gebunden an die Epithelzellen des Duodenums, sie umfasst aber eine grössere Strecke derselben, während die Epithelzellen der übrigen Darmtheile nur diffuse Reaction aufweisen. Die Phagocyten sind durchgängig dichter mit $\mathrm{Fe}$-haltigen Körnchen besetzt und zeigen auch eine diffuse Durchtränkung.

Ausserdem konnte eine Proliferation der Leukocyten beobachtet werden, mit der eine Hyperämie des Stromas der Zotten Hand in 
Hand ging. Es ist das Bild einer verstärkten Resorptionsthätigkeit, das wir vor uns haben, eine Leukocytose der Darmmucosa, bedingt durch den von den $\mathrm{Fe}$-Präparaten hervorgerufenen Reiz. Da die Thiere bei der Tödtung unter durchaus günstigen Ernährungsverhältnissen sich befanden, so muss man annehmen, dass wir es hier nicht mit pathologischen Verbältnissen zu thun haben. Diese Annahme wird noch dureh den Umstand unterstützt, dass an dem Thiere B, das nach einer Fütterung von 20 Tagen mit $75 \mathrm{mg}$ Fisen $1 \times 24$ Stunden hungerte, das Fpithel vollkommen frei von jeglicher Reaction war und sich in Nichts von dem sonst $1 \times 24$ Stunden gehungert habenden untersehied.

Das Object $C$, das in 15 Tagen $180 \mathrm{mg}$ Fe erhalten hatte, bot uns endlich im Jejunum und Ileum ein Bild der Resorption, das uns gestattet, dem Wege des Eisens aus dem Darmcanale in die Zotte zu folgen. Jetzt müssen wir uns die Frage vorlegen, ob die in den Lieberkühn'schen Krypten des Coecums nachgewiesenen Fe-Reactionen der Ausscheidung oder Aufnahme zuzuschreiben sind. Wir fanden bei dem normalen ${ }^{1}$ ), dann bei dem mit Fe-armer Nahrung gefütterten Thiere Fo-Reaction anfweisende Mucinpfrönfe im Coecum, und schliesslich konnten wir bei dem mit ferr. oxyd. saceh. solub. behandelten Thiere Aehnliches aufweisen. Der Unterschied zwischen den einzelnen Erscheinungen der Ausscheidung bestand darin, dass bei dem normalen, wie auch bei dem mit Fe-armer Nahrung ernährten Thiere die Pfröpfe aus fester Masse gebildet waren und nicht aus flüssiger, wie es bei den mit $\mathrm{Fe}$ gefütterten Thieren der Fall war.

Samojloff ${ }^{2}$ ) fand bei einer Katze, der er $126 \mathrm{mg} \mathrm{Fe}$ in Form von Hornemann'scher Zuckereisenlösung in die Vena jugul. injicirte, nachdem sie 14 Tage darauf eingegangen war, in den Lumina der Lieberkühn'schen Krypten des oberen Dünndarms eine blaue Masse, die er für ausgeschiedenes $\mathrm{Fe}$ ansieht. $\mathrm{Macall \textrm {um } ^ { 3 }}$ ) fand nach grossen Fe-Gaben, besonders nach Fe-Pcpton per os eine sehr intensive Fe-Reaction in den Lieberkühn'schen Drüsen des Dünndarms des Coecums und des oberen Dickdarms der Meerschweinchen. Die Drüsenzellen selbst gaben ihm nie Fe-Reaction. Er sieht den Vorgang für eine Ausscheidung an und erklärt die Abwesenheit der Fe-Reaction

1) cf. Tafel VI Fig. 1 u. 2.

2) Samojloff, Arbeiten des pharmakologischen Instituts zu Dorpat Bd. 9 S. 13. 1893 .

3) Macallum, 1. c. S. 278. 
in den Drüsenzellen dadurch, dass er annimmt, die jeweilig ausgeschiedenen $\mathrm{Fe}$-Mengen wären sehr gering und die Secretionsgeschwindigkeit so gross, dass eine Fe-Reaction nicht nachweisbar sei.

Hochbaus und Quincke ${ }^{1}$ ) fanden in den Lieberkühn'schen Krypten des Dickdarmes von Mäusen, die mit Fe gefüttert waren, eine Menge von intensiver Fe-Reaction, die sie nicht bestimmt zu deuten wagen. Sie sagen: „es könnte von aussen eingedrungener Fe-haltiger Darminbalt sein, es könnte das $\mathrm{Fe}$ durch ausgewanderte Leukocyten erst dahin gelangt sein. " In die Drüsen gelangter Darminhalt ist die Fe-haltige Masse in den Lieberkühn'schen Krypten keineswegs; ebensowenig gelang es mir nachzuweisen, dass Fe-Cyten in die Drüse hiıeinwanderten, während Leukocyten oft bei der Einwanderung anzutreffen waren.

Wenn man sich vorstellt, dass die im interglandulären Gewebe reichlich vorhandenen Fe-Cyten oder auch das Plasma der Capillaren ihr Fe den Drüsenzellen abgeben, so müsste dasselbe von ihnen in eine feste Form umgewandelt und dann wieder in lockerer Form ausgeschieden werden. Diese Anschaumg hat viel Bestechendes in Anbetracht dessen, dass das Fe als Sauerstoffüberträger im thierischen Organismus unzweifelhaft eine wesentliche Rolle spielt, zumal bei den verwickelten Processen in den Drüsen. Allein das Mucin selbst enthält kein $\mathrm{Fe}$, und in den Analysen der von den verschiedensten Mucinarten abstammenden Aschen konnte ich keine Angabe über das Fe finden. Da sich aber die Gegenwart des letzteren in den Mucinpfröpfen auf eine andere Art erklären lässt, möchte ich es versuchen, hierauf Bezügliches im Nachstehenden anzuführen.

Durch Mayer ${ }^{2}$ ) ist die Beobachtung gemacht worden, dass Submaxillarismucin, im essigsauren $\mathrm{Fe}$ gequollen, dasselbe aufnimmt und sehr festhält. $\mathrm{List}^{3}$ ) machte die Wahrnehmung, dass selbst bei der einfachen Behandlung mit Blutlaugensalz, wo also keine Spur von $\mathrm{Fe}$ in das Präparat gebracht wird, sondern einzig und allein durch Umsetzung (bewirkt durch die Salzsäure) die Ferrocyanwasserstoffsäure gebildet wird, woraus durch den Sauerstoff der Luft Berlinerblau entsteht, der im Darmlumen liegende freie Schleim sich stark

1) Hochhaus und Quincke, 1. c. S. 169.

2) Mayer, Ueber Schleimfärbung. Mittheilungen aus der zoolog. Station zu Neapel. Bd. 12 Heft 2 S. 326. 1896.

3) Th. List, Beiträge zur Chemie der Zellen und Gewebe. Mittheilungen aus der zoolog. Station zu Neapel. Bd. 12 Heft 3 S. 489. 1896. 
Ueber die Resorption und Ausscheidung des Eisens im Darmcanale etc. 505 blau färbte. Die Schleimdrüsen des Darmes zeigten keine Spur einer Blaufärbung.

Wir sehen, dass das Mucin eine grosse Affinität zum Fe hat, und wissen, dass im Darminhalte bei Fe-Fütterung stets Fe vorhanden ist. Man hätte sich hierbei vorzustellen, dass das in den Krypten gebildete Mucin Zeit gehabt hat, $\mathrm{Fe}$ aus dem Darminhalte an sich zu ziehen, bevor noch der Darm aufgeschnitten und in die fixirende Flüssigkeit gelegt worden. Die Mucinpfröpfe scheinen längere Zeit in dem Lumen der Drüsen sich zu befinden, bevor sie ausgestossen werden. Ist das Secret flüssiger Art, so sind die Ränder der Ausmündung der Drüse, also das Oberfächenepithel, häufig benetzt von der secernirten mucinhaltigen Flüssigkeit, die daselbst adhärirt und Fe-Reaction aufweist. Dass das zum Darmlumen hin gerichtete Ende der Pfröpfe so ganz besonders stark reagirt und ziemlich schnell die Abnahme der Fe-Reaction zum Fundus hin statt hat, würde gerade für die Aufnahme des $\mathrm{Fe}$ aus dem Darmlumen sprechen, indem das zum letzteren hingewandte Ende des Pfropfes am längsten mit ihm in Berührung gekommen ist. Unter gewöhnlichen Verhältnissen hat das Meerschweinchen genug Gelegenheit, Fe-Verbindungen mit der Nahrung aufzunehmen; schwieriger ist es zu erklären, warum die mit Fe-armer Nahrung gefütterten Thiere solche Fe-haltige Pfröpfe ausscheiden. Wir müssen uns aber daran erinnern, dass im Dünndarme dieser Thiere eine bedeutende Auswanderung von Fe-Cyten nachzuweisen, mithin dem Kothe $\mathrm{Fe}$ in lockerer organischer Verbindung zugeführt war. Ausserdem wissen wir nicht genau, wie lange der Koth im Coecum verbleibt; es kann daher in ihm noch ein solcher vorhanden sein, der aus der Zeit stammte, wo das Thier normal ernährt wurde, also Möglichkeit hatte, gelegentlich anorganische Fe-Verbindungen in sich aufzunehmen.

\section{Schluss.}

Fassen wir, an das Ende unserer Arbeit angelangt, zusammen, was aus derselben für die Aufnahme und Ausscheidung des Eisens im Darmcanale der Meerschweinchen sich ergiebt, so finden wir Folgendes:

Wir begegnen der Fe-Reaction im Allgemeinen im ganzen Darmcanale, mit Ausnahme des Magens. Diese Fe-Reaction äussert sich am Epithel, dem adenoiden Bindegewebe und dessen Zellen, wie auch an den Blutgefässen. 
Was zunächst das Epithel betrifft, so finden wir bei normalen Thieren, speciell im Duodenum, eine Form des Auftretens von $\mathrm{Fe}$ in den Epithelzellen, der wir an keiner weiteren Partie des Darmcanales begegnen - eine feinkörnige Ablagerung von Fe-Partikeln in dem äusseren, dem Darmlumen zugewandten Viertel der Zelle. Aus diesem Befunde schliessen Hall, Hochhaus und Quincke, wie auch Gaule, dass das Duodenum die einzige Stelle für die Resorption des Nahrungs- wie medicamentös zugeführten Eisens sei.

Der Grund für diese anscheinende Exclusivität des Duodenums scheint mir darin zu liegen, dass letzteres der erste Fe-resorbirende Darmtheil ist und nicht in einem specifischen Vorzuge desselben; es ist der Ueberschuss des $\mathrm{Fe}$, der sich feinkörnig in der Zelle niederschlägt. Einen ähnlichen Vorgang konnten wir in der Leber beobachten, wenn die Fütterung mit Fe längere Zeit gewährt hatte:

Die peripheren Drüsenzellen der Läppchen, welche früher die Zufuhr des Fe aus den Portalgefässen erhielten, wiesen auch immer eher die feinkörnige Ablagerung auf, die dann bei weiterer Zufuhr des Eisens centralwärts vorrückte, nachdem ein Stadium der diffusen Reaction vorhergegangen war. Uebrigens zeigen auch nicht alle Duodenalzellen diese Einlagerung; an vielen Zotten desselben Darmtheiles ist dann nur eine diffuse Fe-Reaction wahrzunehmen, die uns somit als zweite Form, in welcher das resorbirte Fe in den Epithelzellen sich manifestirt, entgegentritt. Diese Form finden wir im ganzen Dünndarm, wie auch im Coecum, bei Fe-Fütterung, während bei normalen Thieren nur in den Duodenalzellen und in einem Falle im Coecum Fe-Reaction an den Epithelien sich nachweisen liess.

Da ich die Fe-Reaction in den Epithelzellen des Coecums als Resorptionsvorgang ansehe, so will ich im Folgenden die Gründe hierfür angeben.

Wie ich im Capitel über die Untersuchungsmethoden hervorgehoben habe, wurden die durch die Berlinerblau-Reaction gewonnenen Schnitte stets durch die Schwefelammonium-Reaction controlirt, so dass mir der Vorwurf der Einseitigkeit nicht gemacht werden kann.

Ausserdem wurde das Coecum des Thieres $A$, das mit Fe gefüttert worden, in der Narkose entnommen und sofort mit physiologischer Kochsalzlösung abgespült, so dass die Fäces nur ganz kurze Zeit ausserhalb des Körpers mit dem Darmepithel in Berührung blieben. Nun kann ja der Einwand gemacht werden, dass die in den Epithelzellen vorhandene Fe-Reaction von dem Fe stamme, das 
von den im adenoiden Gewebe in bedeutender Anzahl vorhandenen $\mathrm{Fe}$. Cyten ausgeschieden würde, was ich andeutungsweise im Duodenum des Frosches gefunden und anfangs dieser Arbeit beschrieben habe. Allein ich muss bemerken, dass die Art und Weise der Vertheilung des Eisens in der Epithelzelle des Frosches eine ganz andere ist. Man überzeugt sich bald davon, dass der Theil der Zelle, welcher den mit Eisen angefüllten Interstitien der Zellen am nächsten liegt, auch eine stärkere Reaction aufweist. In Coecumepithel des betreffenden Meerschweinchens ist die Reaction gleichmässig über die ganze Zelle vertheilt und differirt durchaus nicht mit der Fe-Reaction in den Epithelzellen der Zotten des Jejunums und Ileums desselven Thieres. Hinzufügen möchte ich noch, dass im Coecumepithel der Hungerthiere, in welchem durch die trichterförmigen Canäle eine Emigration von $\mathrm{Fe}$-Cyten zu beobachten war, die Epithelzellen selbst nirgends eine Fe-Reaction aufwiesen.

Die Wege der Aufnahme des Fe lassen sich am besten verfolgen, wenn man die Bcobachtungen, an den mit Fe gefütterten Thieren gewonnen, der Betrachtung zu Grunde legt. Wir finden dann Folgendes:

Durch die Epithelzellen der Zotten, die zum Theil in unmittelbarem Zusammenhang mit den Blutgefässen stchen, wie auch durch die Zwischenräume der Epithelien, geht das $\mathrm{Fe}$ in die Blutgefüsse des Stromas der Zotten des Dündarmes, resp. des interglandulären Gewebes des Coecums über. In den Blutgefässen, wo das Fe an das Plasma gebunden ist, wird es von den Plagocyten aufgenommen, mit denen es dann den Wog zur Leber antritt. Ein anderer Thoil wird aus dem Stroma in locker gebundener Form dem centralen Chyluskanal ubermittelt, ohne dass Fe-Cyten selbst in den letzteren hincingelangen und begibt sich längs der Lymphbahn durch den Ductus thoracicus in den Blutkreisłauf. Z wischen den mit Fe gefütterten und dem mit gewöhnlichem Futter ernährten Thiere konnte ich nur einen quantitativen Unterschied in der Resorption nachweisen.

In Betreff dor Ausscheidung des $\mathrm{Fe}$ ist es uns beim normalen Thiere nicht gelungen, eine Emigration der Fe-Cyten durch das Epithel des Darmeanals zu constatirea, während kleine runde Wanderzellen, die mikrochemisch aber keine Fe-Reaction gaben, in grosser Menge zum Darmlumen hin sich begaben.

Für die Fe-Frage ist von grossem Interesse der Umstand, dass 
die Fe-haltigen Leukocyten oder Phagocyten, die wir, der Kürze halber, einfach "Fe-Cyten" genannt haben, von den neueren Autoren wie Barker ${ }^{1}$ ), Przewoski ${ }^{2}$ ), Teichmüller ${ }^{3}$ ), als eosinophile Zellen angesehen werden. Teichmüller fand, dass dịe Phagocyten der normalen und hungernden Meersehweinchen deutliche Eosinophilie aufweisen.

Auch fand der zuletzt genannte Autor beim hungernden Thiere eine beträchtliche Abnahme der Zellen, während beim normal gefütterten, im besten Wohlsein getödteten Thiere das Vorhandensein der eosinophilen Zellen reichlich war. Diese Beobachtnng ist auch durch meine Untersuchungen bestätigt und noch dahin erweitert worden, dass beim Hunger mit Maulkorb die Phagocyten im Dünndarme vollkommen schwinden können.

Mit dem Schwunde der Phagocyten geht eine Atrophie des adenoiden Gewebes Hand in Hand. Wenn man diesen Zustand des Stromas der Zotte mit demjenigen vergleicht, wie er bei den mit $\mathrm{Fe}$ gefütterten Thieren uns entgegentritt, so fällt der Unterschied ganz besonders auf. In dem einen Falle atrophische Zotte und Schwund der Phagocyten, im andern ein strotzend mit letzteren angefülltes Stroma.

Die entsprechendste Deutung dieses Factums ist, meiner Ansicht nach die, dass in Folge des ausfallenden Reizes von Seiten des Darminhaltes auf die Mucosa die Thätigkeit der letzteren herabgesetzt wird und schliesslich ganz sistirt. Auf den Umstand, dass die adenoide Substanz der Mucosa selbst schwindet, ist ganz besonderes Gewicht zu legen, da hierdurch für die Anschauung, dass die eosinophilen Zellen, zu einem Theile wenigstens, aus der Darmmucosa stammen, an Boden gewonnen wird. Dass die Mucosa des Darmes nicht die ausschliessliche Quelle für die eosinophilen Zellen zu sein scheint, geht aus der Beobachtung Teichmüller's hervor, dass in dem Respirationstractus der normalen Meerschweinchen eine viel

1) Barker, On the presence of iron in the granules of the eosinophile leukocytes. Bull. of the John Hopkins Hosp. Baltimore 1894.

2) Przewoski, Ueber die locale Eosinophilie beim Krebs nebst Bemerkungen über die Bedeutung der eosinophilen Zellen im Allgemeinen. Centralbl. für Path. Bd. 2 Nr. 5. 1896.

3) W. Teichmüller, Das Vorkommen und die Bedeutung der eosinophilen Zellen im Sputum. Deutsch. Arch. f. klin. Med. Bd. 60 Heft 6. 1898. 
Ueber die Resorption und Ausscheidung des Eisens im Darmcanale etc. 509 grössere Menge eosinophiler Zellen zu finden waren, als in der Darmschleimhaut. Er ist daher geneigt, die Lungen und Bronchien für die locale Bildungsstätte der eosinophilen Zellen anzusehen. Wenn auch die Entscheidung dieser Frage von weiteren Untersuchungen abhängen wird, so ist für die Fe-Frage von Interesse der Nachweis Teichmüller's, dass in dem durch leichte Abschabung gewonnenen Schleime der Trachea und der Bronchien zahlreiche eosinophile Zellen vorhanden waren.

Da letztere Fe-haltig sind, ist anzunehmen, dass normaler Weise eine Ausscheidung des Fe auf der Respirationsschleimhaut der Meerschweinchen stattfinciet, was neue Gesichtspunkte für die Frage nach der Bedeutung des Fe im normalen Organismus eröffnet.

Die Anschauung, dass den eosinophilen Zellen eine hohe Bedeutung bei dem Kampfe des Organismus mit den ihn bedrohenden Schädlichkeiten zukommt, findet auch durch meine Untersuchungen eine Bestätigung. Bei den Hungermeerschweinchen fanden wir in bedeutender Menge Fe-Cyten und erst in späteren Stadien Erythrocyten sowohl durch die trichterförmigen Canäle des Obertlächenepithels des Coecums, wie auch durch die des Dickdarms emigriren; es fand sich das aber nur bei den ohne Maulkorb hungernden Thieren. Die mit Maulkorb hungernden Meerschweinchen befanden sich somit in ungünstigeren Bedingungen in Bezug auf den Kampf mit den durch den Hunger hervorgerufenen Zersetzungsproducten des Körpers.

Aehnliches fand 'Teichmüller bei Phthisikern: Zu Zeiten, wo der Organismus erfolgreich mit dem Bakteriengifte kämpfte, waren auf der Respirationsschleimhant eosinophile Zellen in grosser Anzahl, die zu anderer Zeit fehlten.

Bei den mit Fe gefütterten Thieren fanden wir, dass die Ausscheidung desselben vor sich ging, wo eine Quantität aufgenommen war, die für den Organismus eine Grenze des Aufnehmbaren bedeutete. Das Versuchsthier $C$ hatte $180 \mathrm{mg}$ met. Fe in 15 Tagen erhalten, die grösste Quantität, die überhaupt meinen Versuchsthieren per os zugeführt worden und wir konnten im oberen und unteren D i ckd a rm unzweideutige Bilder der Ausscheidung des Fe auffinden. Der Organismus war also auf nen Punkt angelangt, wo er sich von dem Ueberschuss befreien musste. Dies geschah durch Fe-haltige Leukocyten kleiner Form. 
510 G. Swirski: Ueber die Resorption und Ausscheidung des Eisens etc.

In wie weit die einzelnen Zellformen an den verschiedenen Aufgaben im Dienste des Organismus sich betheiligen, kann erst durch weitere mühsame Untersuchungen festgestellt werden.

\section{Beschreibung der Zeichnungen.}

Fig. 1. stellt eine Falte des Coecums vom normalen Meerschweinchen I dar. Man sieht die in den Lieberkühn'schen Krypten befindlichen und zum Darmlumen hin gerichteten blaugefärbten Mucinpfröpfe. Zeichenocular Leitz, Object. 3.

Fig. 2. Das vorige bei stärkerer Vergrösserung. Zeichenocular Leitz, Object. 7. Fig. 3. Zotte aus dem Jejunum des mit Eisensaccharat gefütterten Meerschweinchens $C$. In den Blutgefässen sind die Erythrocyten gelb gemalt worden. Zeichenocular Leitz, Object. 7. 
ig. 3
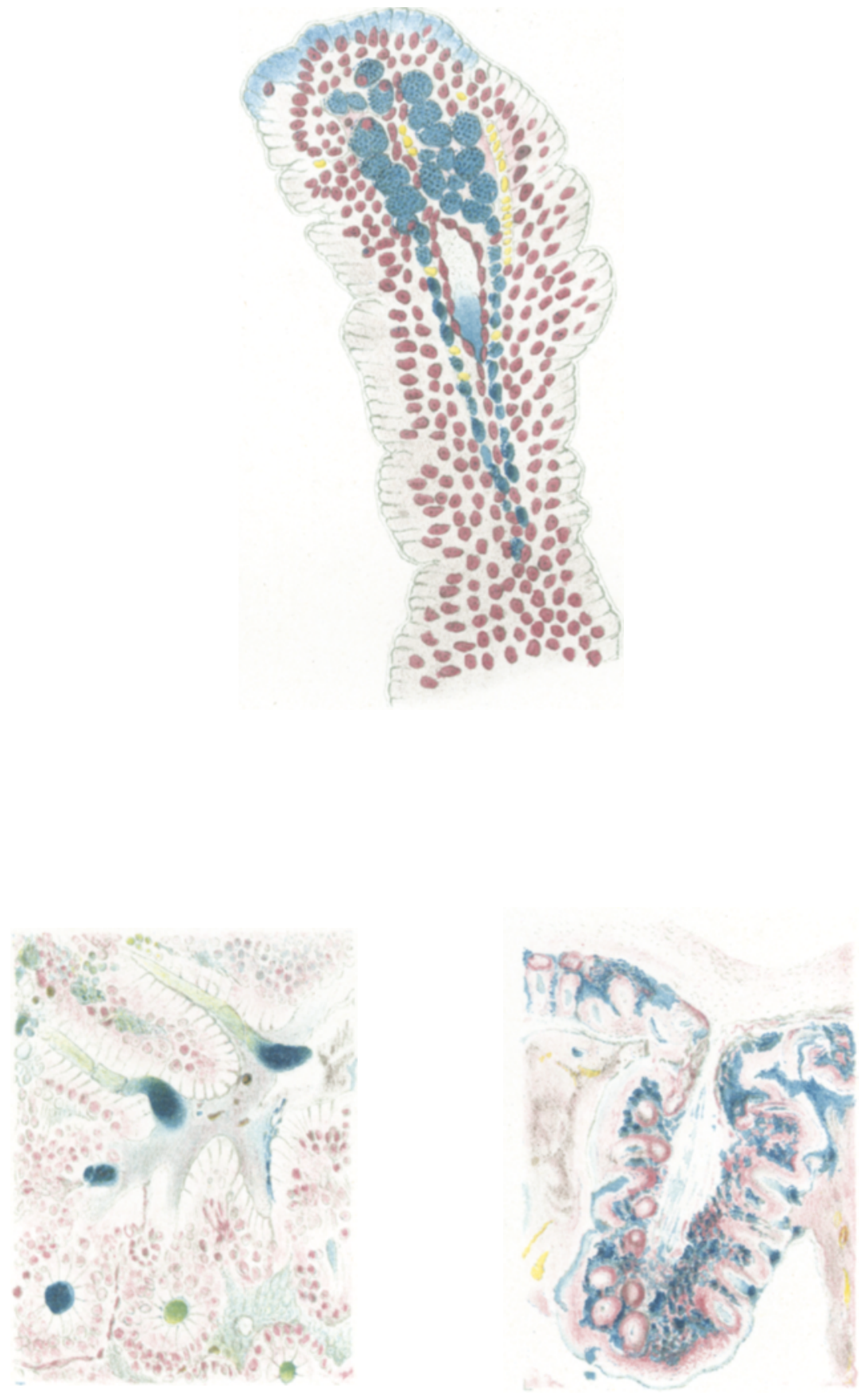